\title{
ABSORPTION FILAMENTS TOWARD THE MASSIVE CLUMP G0.253+0.016
}

\author{
John Bally ${ }^{1}$, J. M. Rathborne ${ }^{2}$, S. N. Longmore ${ }^{3}$, J. M. Jackson ${ }^{4}$, J. F. Alves ${ }^{5}$, E. Bressert ${ }^{6}$, Y. Contreras ${ }^{6}$, J. B. Foster $^{7}$,

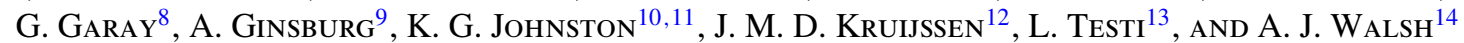 \\ ${ }^{1}$ Department of Astrophysical and Planetary Sciences, University of Colorado, UCB 389, Boulder, CO 80309 USA; John.Bally@ colorado.edu \\ ${ }^{2}$ CSIRO Astronomy and Space Science, P.O. Box 76, Epping NSW 1710, Australia; Jill.Rathborne@ @siro.au \\ ${ }^{3}$ Liverpool Johns Moore University, Liverpool, UK \\ ${ }^{4}$ Institute for Astrophysical Research, Boston University, Boston, MA 02215, USA; jackson@bu.edu \\ ${ }^{5}$ University of Vienna, Turkenschanzstrasse 17, A-1180 Vienna, Austria; joao.alves@univie.ac.at \\ ${ }^{6}$ CSIRO Astronomy and Space Science, P.O. Box 76, Epping NSW 1710, Australia; ebressert@ gmail.com, Yanett.Contreras@csiro.au \\ 7 Yale Center for Astronomy and Astrophysics, Yale University, New Haven, CT 06520, USA; jonathan.b.foster@yale.edu \\ ${ }^{8}$ Universidad de Chile, Camino El Observatorio 515, Las Condes, Santiago, Chile; guido@ das.uchile.cl \\ ${ }^{9}$ European Southern Observatory, Karl-Schwarzschild-Strasse 2, D-85748 Garching bei Munchen, Germany; adam.g.ginsburg@ gmail.com \\ ${ }^{10}$ Max Planck Institute for Astronomy, Koenigstuhl 17, Heidelberg D-69117, Germany; johnston@ @mia-hd.mpg.de \\ ${ }^{11}$ School of Physics and Astronomy, University of Leeds, Leeds LS2 9JT, UK \\ ${ }^{12}$ Max Planck Institut fur Astrophysik, Karl-Schwarzschild-Strasse 2, D-85740 Garching bei Munchen, Germany; kruijssen@mpa-garching.mpg.de \\ ${ }^{13}$ European Southern Observatory, Karl-Schwarzschild-Strasse 2, D-85748 Garching bei Munchen, Germany; 1testi@eso.org \\ ${ }^{14}$ International Centre for Radio Astronomy Research, Curtin University, GPO Box U1987 Perth, Australia; andrew.walsh@ curtin.edu.au \\ Received 2014 April 27; accepted 2014 September 5; published 2014 October 9
}

\begin{abstract}
ALMA $\mathrm{HCO}^{+}$observations of the infrared dark cloud G0.253+0.016 located in the central molecular zone of the Galaxy are presented. The $89 \mathrm{GHz}$ emission is area-filling, optically thick, and sub-thermally excited. Two types of filaments are seen in absorption against the $\mathrm{HCO}^{+}$emission. Broad-line absorption filaments (BLAs) have widths of less than a few arcseconds (0.07-0.14 pc), lengths of 30-50 arcsec (1.2-1.8 pc), and absorption profiles extending over a velocity range larger than $20 \mathrm{~km} \mathrm{~s}^{-1}$. The BLAs are nearly parallel to the nearby G0.18 non-thermal filaments and may trace $\mathrm{HCO}^{+}$molecules gyrating about highly ordered magnetic fields located in front of G0.253+0.016 or edge-on sheets formed behind supersonic shocks propagating orthogonal to our line of sight in the foreground. Narrow-line absorption filaments (NLAs) have line widths less than $20 \mathrm{~km} \mathrm{~s}^{-1}$. Some NLAs are also seen in absorption in other species with high optical depth, such as HCN, and occasionally in emission where the background is faint. The NLAs, which also trace low-density, sub-thermally excited $\mathrm{HCO}^{+}$molecules, are mostly seen on the blueshifted side of the emission from G0.253+0.016. If associated with the surface of G0.253+0.016, the kinematics of the NLAs indicate that the cloud surface is expanding. The decompression of entrained, milli-Gauss magnetic fields may be responsible for the re-expansion of the surface layers of G0.253+0.016 as it recedes from the Galactic center following a close encounter with Sgr A.
\end{abstract}

Key words: Galaxy: center - ISM: clouds - ISM: individual (G0.253+0.016) - stars: formation

Online-only material: color figures

\section{INTRODUCTION}

The central molecular zone (CMZ) at Galactocentric radii less than $\sim 500 \mathrm{pc}$ contains the most massive, dense, and turbulent molecular clouds in the Galaxy (Rodriguez-Fernandez \& Combes 2008; Ferrière et al. 2007; Rodriguez-Fernandez et al. 2006; Pierce-Price et al. 2000; Morris \& Serabyn 1996; Bally et al. 1987b, 1988) along with some of the most compact and massive star clusters (Figer et al. 2002; Habibi et al. 2013).

Lis \& Carlstrom (1994) found an exceptionally massive and dense cloud seen in projection against the bright near- and mid-infrared emission from the CMZ, G0.253+0.016. Located toward the brightest portion of the Galactic plane at infrared wavelengths, this cloud was clearly seen in silhouette against the $\mathrm{CMZ}$ in the IRAS mid-infrared images and is the most prominent infrared dark cloud (IRDC) in the sky (Menten et al. 2005; Arendt et al. 2008; Ramírez et al. 2008).

Lis et al. (1994) and Lis \& Menten (1998) demonstrated that this cloud has an unusually high mass $\left(M>10^{5} M_{\odot}\right)$ and density $\left(N\left(\mathrm{H}_{2}\right)>10^{5} \mathrm{~cm}^{-2}\right)$ and is very compact $(r<3 \mathrm{pc})$. Lis et al. (2001) used Infrared Space Observatory (ISO) data and millimeter-wave spectra to show that the dust temperature is low but the gas is hot. In the cloud center, $15<T_{\text {dust }}<$
$22 \mathrm{~K}$ but increases to $\sim 27 \mathrm{~K}$ at the cloud edges (Longmore et al. 2012). On the other hand, the gas is hot with $T_{\text {gas }} \sim$ $80 \mathrm{~K}$ (Ao et al. 2013). The mid-infrared fine-structure cooling lines measured with $I S O$ indicated that near-UV interstellar radiation field surrounding the cloud is about $10^{3}$ times stronger than in the Solar vicinity (Lis et al. 2001), consistent with warmer dust at the cloud periphery. Kauffmann et al. (2013) presented interferometric $\mathrm{SiO}$ and $\mathrm{N}_{2} \mathrm{H}^{+}$maps of $\mathrm{G} 0.253+0.01$ that exhibit some localized narrow spectral lines with central velocities covering the wide velocity range seen in single-dish data (Rathborne et al. 2014b). The large line widths and bright emission in tracers such as $\mathrm{SiO}$, and the complicated kinematics was interpreted as evidence for shocks, possibly indicating an early stage of a cloud-cloud collision (Higuchi et al. 2014).

G0.253+0.016 (also known as the "Lima Bean" or the "Brick") has been proposed to be a possible progenitor to a massive star cluster (Longmore et al. 2012). Fitting the dust continuum spectral energy distribution to the Herschel 60, 160, 250, 350, and $500 \mu \mathrm{m}$ data obtained from the Herschel Space Observatory Hi-GAL survey (Molinari et al. 2011, 2010a, 2010b) and $1100 \mu \mathrm{m}$ from the Bolocam Galactic Plane Survey (Bally et al. 2010) reveals a dust temperature of $\sim 20 \mathrm{~K}, \mathrm{H}_{2}$ column density of $\sim 3.3 \times 10^{23} \mathrm{~cm}^{-2}$, and mass of $\sim 10^{5} M_{\odot}$ 
(Longmore et al. 2012, 2013). Absorption in the near- and farinfrared and application of the virial theorem to spectral line data (Rathborne et al. 2014b) give similar results for the mass and column density. With a mean radius of about $3 \mathrm{pc}$ and a mean density $n\left(\mathrm{H}_{2}\right) \approx 7 \times 10^{4} \mathrm{~cm}^{-3}, \mathrm{G} 0.253+0.016$ has sufficient mass to potentially form a young massive cluster (PortegiesZwart et al. 2010). However, previous studies found no evidence for internal heating sources or embedded $\mathrm{H}$ II regions (Lis et al. 2001). Only one $\mathrm{H}_{2} \mathrm{O}$ maser has been found in $\mathrm{G} 0.253+0.016$ (Lis et al. 1994; Breen \& Ellingsen 2011). Thus, G0.253+0.016 may either be in the very earliest stages of high-mass star or cluster formation, or because of the extreme conditions in the CMZ, may fail to form many stars.

Most star formation in the CMZ occurs on a twisted elliptical ring-like structure with a projected radius of about $100 \mathrm{pc}$ and offset toward positive Galactic longitudes with respect to the dynamical center of the Galaxy (Rodriguez-Fernandez et al. 2006; Molinari et al. 2011). The ring contains the Sgr C, Sgr A (which contains the supermassive black hole marking the center of the Galaxy), Sgr B1, and Sgr B2 star forming complexes. G0.253+0.016 is the most prominent member of a chain of IRDCs at positive Galactic longitudes between Sgr A and Sgr B2 (Lis et al. 2001; Longmore et al. 2013). Longmore et al. (2012) and Rathborne et al. (2014b) found that G0.253+0.016 exhibits a line width near zero intensity of about $\sim 50-60 \mathrm{~km} \mathrm{~s}^{-1}$, and an internal velocity dispersion of at least $16 \mathrm{~km} \mathrm{~s}^{-1}$ typical for CMZ clouds that implies a crossing time of about $0.17 \mathrm{Myr}$.

Models of orbits in barred, tri-axial potentials indicate that gas in the inner CMZ is likely to be moving on $x_{2}$ orbits elongated along the minor axis of the bar (Contopoulos \& Papayannopoulos 1980; Athanassoula 1992a, 1992b). The major axis of the bar is oriented between $20^{\circ}$ and $45^{\circ}$ with respect to our line of sight (Binney et al. 1991; Rodriguez-Fernandez \& Combes 2008). G0.253+0.016 is located at a projected distance of about $50 \mathrm{pc}$ from $\mathrm{Sgr} \mathrm{A}$ and has a radial velocity $V_{\mathrm{LSR}} \approx$ $40 \mathrm{~km} \mathrm{~s}^{-1}$. Assuming that it is moving close to the plane of the sky with a velocity of about $150-180 \mathrm{~km} \mathrm{~s}^{-1}$ along an elongated $x_{2}$ orbit (this is the speed expected for a circular orbit given the enclosed mass), it would have passed near Sgr A about 0.25-0.3 Myr ago (J. M. D. Kruijssen et al. 2014, in preparation). Clouds injected onto $x_{2}$ orbits near apocenter with velocities lower than the circular orbit speed at that location tend to plunge deeper into the potential well. Following pericenter passage near Sgr A, they climb out of the potential to a second apocenter located on the opposite side of the nucleus, and on the opposite side of the Galaxy from the first apocenter. Such plunging, elongated orbits precess at rates which depend on the details of the enclosed mass distribution and orbit eccentricity. Longmore et al. (2013) proposed that the encounter of G0.253+0.016 with Sgr A may have compressed this cloud.

In this paper, high-angular resolution, high dynamic range, and sensitive ALMA observations of $J=1-0 \mathrm{HCO}^{+}$are presented that reveal an extensive network of filaments seen in absorption toward G0.253+0.016. The most prominent filament (Figure 1) has an absorption line width of over $30 \mathrm{~km} \mathrm{~s}^{-1}$ and is nearly parallel to the bright, non-thermal radio filaments which cross the Galactic plane at longitude 0.18 a few arc minutes from G0.253+0.016. Dozens of filaments with less than $20 \mathrm{~km} \mathrm{~s}^{-1}$ line widths are predominantly seen on the blueshifted side of the $\mathrm{HCO}^{+}$emission from $\mathrm{G} 0.253+0.016$. In this paper, it is shown that these absorption features are produced by very low density, sub-thermally excited $\mathrm{HCO}^{+}$absorbing optically thick, but nevertheless sub-thermally excited background $\mathrm{HCO}^{+}$ emission from this cloud. If located on the near surface of G0.253+0.016, their blueshifted velocities with respect to the background $\mathrm{HCO}^{+}$emission would indicate that the cloud surface layers are expanding, contrary to the naive expectation of global collapse if it were a progenitor to a massive cluster.

\section{OBSERVATIONS}

Observations presented here were obtained with the Atacama Large Millimeter Array (ALMA) located on the Chajnantor plateau in the Northern Chilean Andes. Full details of the observations and data reduction will be presented in another paper (Rathborne et al. 2014a). A short summary is presented below.

G0.253+0.016 was imaged in the $3 \mathrm{~mm}$ wavelength bands (ALMA band 3) using 25 of ALMA's 12 m diameter antennas. The synthesized beam produced by ALMA at this frequency has a diameter of 1 .' 7 corresponding to a linear scale of $0.07 \mathrm{pc}$ at the $8.5 \mathrm{kpc}$ distance of the CMZ. Because the primary-beam fieldof-view of ALMA is small $\left(\sim 70^{\prime \prime}\right)$ and G0.253+0.016 subtends a $1^{\prime}$ by $3^{\prime}$ region, a mosaic consisting of 13 separate pointings was required. Baselines ranged from 13 to $455 \mathrm{~m}$. G0.253+0.016 was imaged on six separate occasions over the period 2012 July 29-2012 August 1. Each data set was independently calibrated prior to merging. The ALMA correlator was configured to measure the continuum in a $1.875 \mathrm{GHz}$ bandpass, and to observe 10 different molecular emission lines with a resolution of $0.488 \mathrm{MHz}$ (about $1.5 \mathrm{~km} \mathrm{~s}^{-1}$ velocity resolution) covering a velocity range from about $V_{\mathrm{LSR}}=-200 \mathrm{~km} \mathrm{~s}^{-1}$ to about $+240 \mathrm{~km} \mathrm{~s}^{-1}$. Single-dish observations of G0.253+0.016 in the same transitions as observed with ALMA were obtained with the $22 \mathrm{~m}$ Mopra radio telescope in Australia as part of the MALT90 Survey (Foster et al. 2011; Jackson et al. 2013; Foster et al. 2013). The resulting maps were used to restore 0-spacings in the ALMA data in the Fourier domain (Rathborne et al. 2014a).

Final data cubes were gridded to a pixel scale of $0^{\prime \prime} 35$ pixel $^{-} 1$. Because the flux in adjacent channels in the data is correlated, the data were re-sampled to $3.4 \mathrm{~km} \mathrm{~s}^{-1}$ per channel. The rms noise in each frequency channel is about $5 \mathrm{mJy}(0.26 \mathrm{~K})$ in the $1^{\prime \prime} .7$ beam. At the rest frequency of the $\mathrm{HCO}^{+} J=1-0$ transition $(89.1885 \mathrm{GHz})$ a flux of $1 \mathrm{Jy}$ in the $1^{\prime \prime} .7$ diameter beam (or a surface brightness of $1 \mathrm{Jy}$ beam $^{-1}$ ) corresponds to a brightness temperature of $53.5 \mathrm{~K}$. All temperatures and fluxes refer to values above the cosmic microwave background or any other smooth background which are resolved out by ALMA and removed from the single-dish observations by beam-switching.

\section{RESULTS}

The blueshifted emission in $\mathrm{HCO}^{+}$shows an extensive network of curved and linear filamentary structures seen in absorption against the warmer background emission from G0.253+0.016. Figure 1 shows an integrated intensity map of $\mathrm{G} 0.253+0.016$ in the $J=1-0 \mathrm{HCO}^{+}$transition. While the $\mathrm{ALMA} \mathrm{HCO}^{+}$integrated intensity map is dominated by areafilling emission, the optically thinner $\mathrm{H}^{13} \mathrm{CO}^{+}$cubes show bright arcs resembling bows moving toward increasing R.A. (left in the figures). This overall morphology is also seen in other optically thin (or at least thinner than $\mathrm{HCO}^{+}$) tracers such as $\mathrm{HNCO}$, $\mathrm{H}_{2} \mathrm{CS}, \mathrm{SiO}$, and SO (Rathborne et al. 2014a).

Figures 2-9 show individual $3.4 \mathrm{~km} \mathrm{~s}^{-1}$ wide channel maps in $\mathrm{HCO}^{+}$. The yellow arrows mark the location of a pair of 


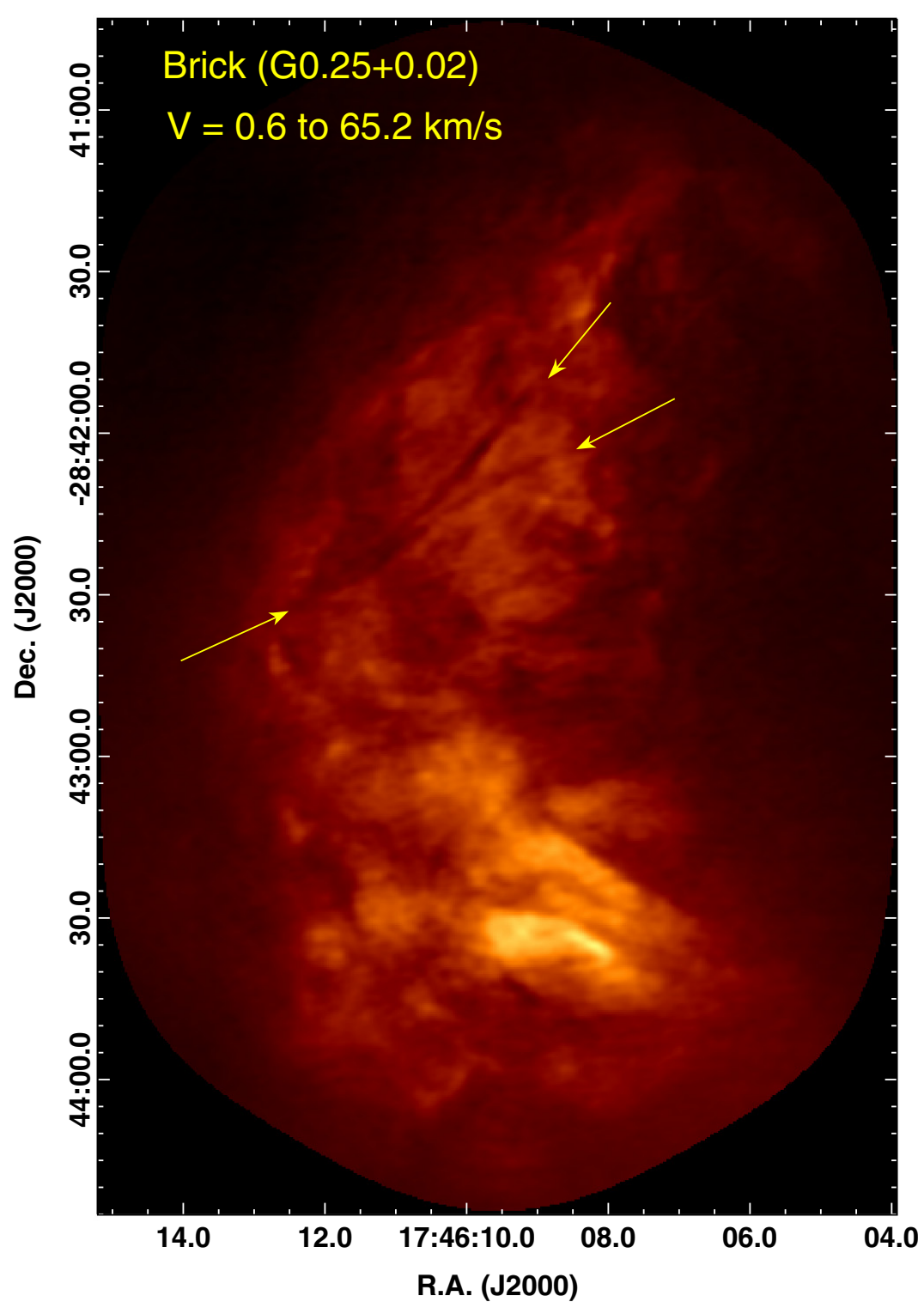

Figure 1. Velocity-integrated image showing $\mathrm{G} 0.253+0.016$ in the $\mathrm{HCO}^{+} J=1-0$ transition. The velocity range extends from $V_{\mathrm{LSR}}=0.6 \mathrm{~km} \mathrm{~s}^{-1}$ to $65.2 \mathrm{~km} \mathrm{~s}^{-1}$. Arrows mark the locations of the two broad-line absorption (BLA) filaments. These arrows are retained in Figures 2-9.

(A color version of this figure is available in the online journal.)

filaments having a length of over $L>60^{\prime \prime}$ and a radial velocity dispersion greater than $20 \mathrm{~km} \mathrm{~s}^{-1}$. Dozens of filaments with line widths less than $10-20 \mathrm{~km} \mathrm{~s}^{-1}$ are located on the blueshifted (low velocity) side of the $\mathrm{HCO}^{+}$emission. These filaments also have widths of only a few arcseconds, but their lengths tend to be shorter than the broad-line filaments. Some filaments form clusters of nearly parallel strands which at extreme blueshifted radial velocities $\left(V_{\mathrm{LSR}}=5-30 \mathrm{~km} \mathrm{~s}^{-1}\right)$ blend into a complex network of absorption covering most of the spatial extent of G0.253+0.016. The morphology of the absorption filaments do not show any correlation with the underlying filamentary structure of G0.253+0.016 seen in optically thin emission lines. The absorption filaments can be subdivided into two categories based on their observed properties:

Broad-line absorption (BLA) filaments have widths of less than a few arcseconds $(<0.04-0.08 \mathrm{pc})$, lengths of 30-50 arcsec $(\sim 1-2 \mathrm{pc})$, and absorption profiles extending over a velocity range larger than $20 \mathrm{~km} \mathrm{~s}^{-1}$ around $V_{\mathrm{LSR}}=10-40 \mathrm{~km} \mathrm{~s}^{-1}$. The two most evident BLAs (marked by in Figures 1-9) are nearly parallel to the nearby G0.18 non-thermal filaments seen in 20 and $6 \mathrm{~cm}$ wavelength radio continuum images of Yusef-Zadeh et al. (2004). These BLAs have position angles (measured from north to east) of P.A. $\sim 125^{\circ}-140^{\circ}$, nearly perpendicular to the Galactic plane. The nearby non-thermal filaments have P.A. $\sim 125^{\circ}$.

Analysis of spatial-velocity cuts and spectra orthogonal to the most prominent BLA filament in Figure 1 shows that it extends from below $17 \mathrm{~km} \mathrm{~s}^{-1}$ to about $45 \mathrm{~km} \mathrm{~s}^{-1}$ (Figure 10). The intensity in the faintest part of the filament ranges from about 0.17 to greater than 0.8 times the $\mathrm{HCO}^{+}$intensity in the surrounding region which has a brightness temperature of $3-4 \mathrm{~K}\left(0.05-0.08 \mathrm{Jy}\right.$ beam $\left.^{-1}\right)$. Thus corresponding brightness temperatures at the most opaque portions of the BLA filaments range from $\sim 0.5 \mathrm{~K}\left(0.01 \mathrm{Jy}^{\text {beam }}{ }^{-1}\right)$ at the darkest part of the main BLA to about $2 \mathrm{~K}\left(0.04 \mathrm{Jy}_{\text {beam }}{ }^{-1}\right)$ at more typical locations. The lowest-opacity occurs near the ends of the 


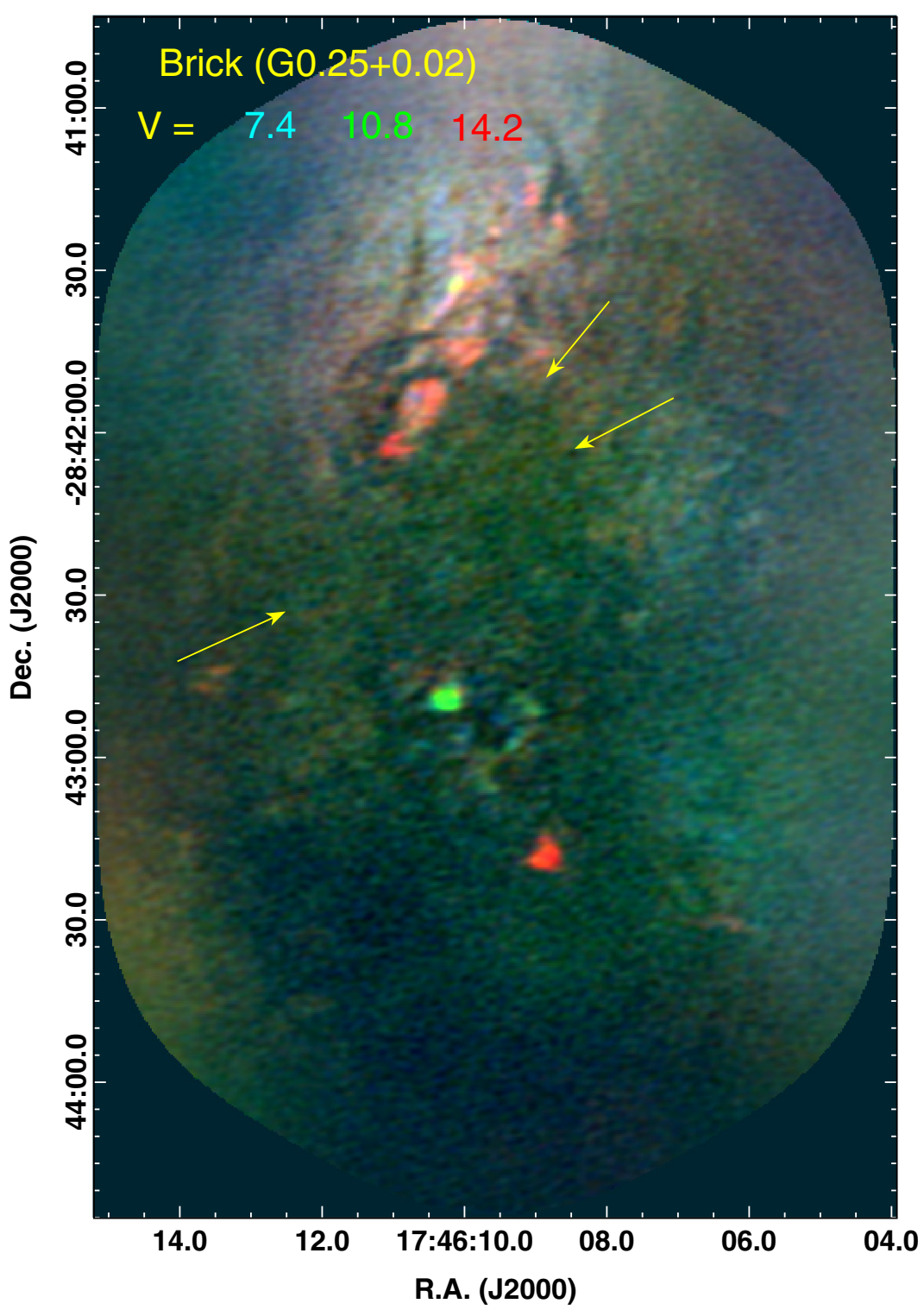

Figure 2. $\mathrm{HCO}^{+}$image of $\mathrm{G} 0.253+0.016$ showing $3.4 \mathrm{~km} \mathrm{~s}^{-1}$ wide channels centered at $V_{\mathrm{LSR}}=7.4$ (blue), 10.8 (green), and 14.2 (red) $\mathrm{km} \mathrm{s}^{-1}$. In addition to the arrows marking the location of the most BLA filaments, the three arrows in the lower right mark the general locations of narrow-line absorption (NLA) filaments in Figures 2-9.

(A color version of this figure is available in the online journal.)

major BLA filaments. The deepest absorption occurs at the redshifted end of the feature near $V_{\mathrm{LSR}} \approx 37 \mathrm{~km} \mathrm{~s}^{-1}$ at $\mathrm{J} 2000 \approx$ 17:46:09.79, -28:42:03.5. The mean flux along cross-cuts is about 0.5 times the surrounding cloud flux. Thus, the optical depth of the absorbing gas ranges from $\sim 0.2$ to about 2 with a mean value of 0.7 . The $\mathrm{HCO}^{+}$BLA filaments have no counterparts in any of the other spectral lines. Specifically, they are not seen in other optically thick species such as HCN.

Narrow-line absorption (NLA) $\mathrm{HCO}^{+}$filaments, some of which are also seen in absorption in other species with high optical depth such as HCN, have line widths of less than $20 \mathrm{~km} \mathrm{~s}^{-1}$. Some tend to cluster into filament bundles which taken together have velocity extents up to $20 \mathrm{~km} \mathrm{~s}^{-1}$. These filaments are mostly located at $V_{\mathrm{LSR}} \sim 10-40 \mathrm{~km} \mathrm{~s}^{-1}$, blueshifted relative to the median radial velocity of $\mathrm{G} 0.253+0.016$.

Table 1 lists several examples of NLA filaments. Near the northwest portion of the cloud, several arcs form nearly concentric loops with radii of about $6^{\prime \prime}$ and $13^{\prime \prime}$ between $V_{\mathrm{LSR}}=$ 11 and $21 \mathrm{~km} \mathrm{~s}^{-1}$ (Figures 2 and 3). NLA 1 is a narrow linewidth absorption feature about $20^{\prime \prime}$ south of the two BLAs. The NLA 2 consists of several parallel east-west strands near the middle of the cloud around $\delta=-28: 42: 50$ (Figures 5-7). NLA 3 is a cluster of bent filaments near the redshifted southern part of $\mathrm{G} 0.253+0.016$ around $\delta=-28: 43: 00$ to $-28: 44: 00$ (Figures 4-6). Many NLA filaments blend into broad regions of absorption on the blueshifted, low-radial velocity side of the $\mathrm{HCO}^{+}$data cube. Figure 11 shows spectra of an NLA filament near the northern side of the NLA 3 cluster. Figure 12 shows the $\mathrm{HCO}^{+}$integrated intensity map of $\mathrm{G} 0.253+0.016$ with the locations of the BLA and NLA filaments with the locations of the features listed in Table 1 marked.

At the extreme blueshifted end of the data cube, the NLA filament network blends into an area-filling absorption. The channel maps between $V_{\mathrm{LSR}}=14$ and $21 \mathrm{~km} \mathrm{~s}^{-1}$ indicate that 


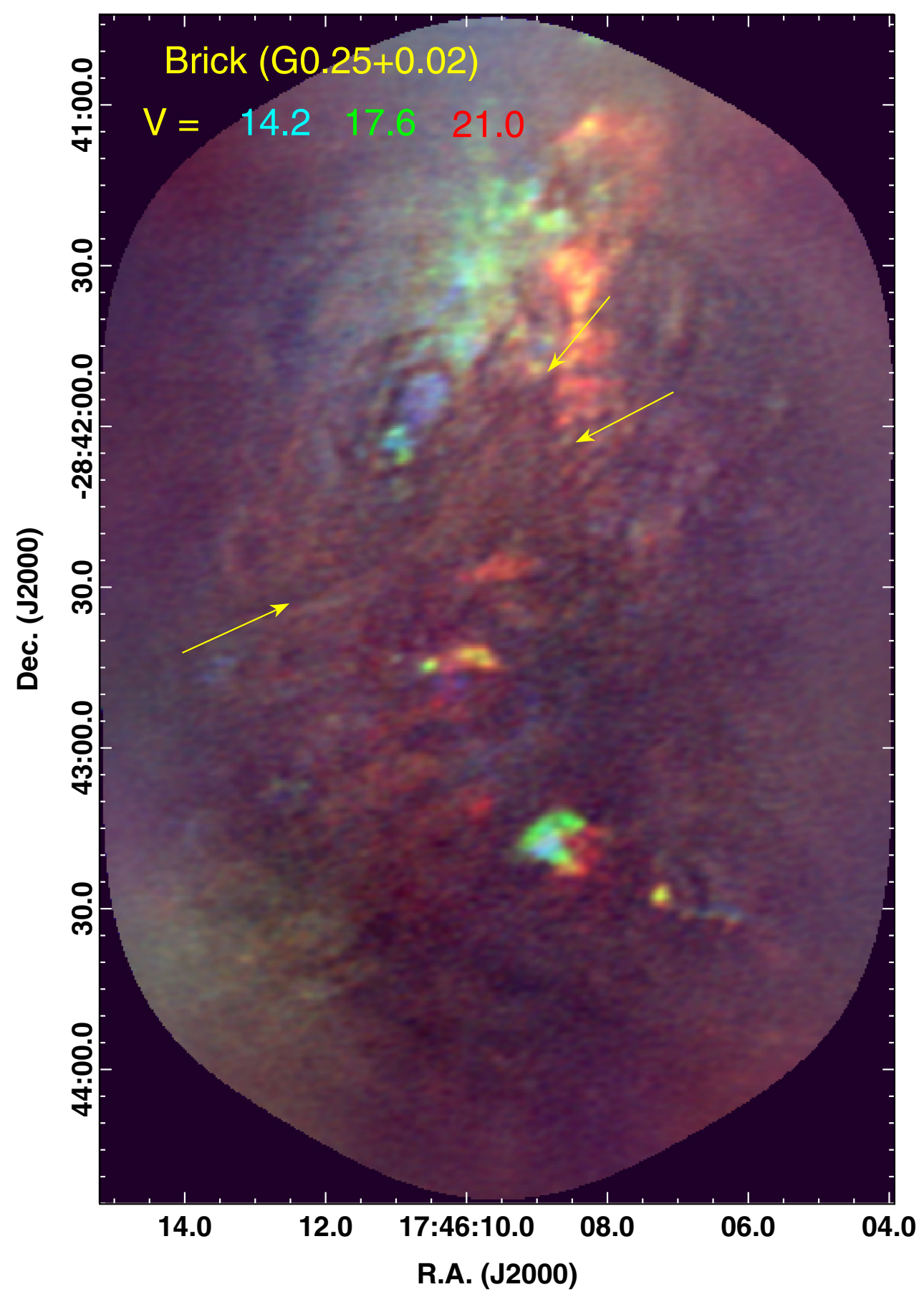

Figure 3. This figure shows $\mathrm{HCO}^{+}$channels centered at $V_{\mathrm{LSR}}=14.2$ (blue), 17.6 (green), and 21.0 (red) $\mathrm{km} \mathrm{s}^{-1}$. (A color version of this figure is available in the online journal.)

most of the surface of G0.253+0.016 is covered by NLA filaments. The fractional area covered diminishes toward higher radial velocities. Most NLAs are shorter than the BLAs and many are curved. A few, such as those centered near J2000 = 17:46:10.6, -28:41:55 trace nearly complete circles. Others are partial arcs. The distinction between BLA and NLA filaments is not sharp; they form a continuum with decreasing line widths and lengths from BLA1 and 2 (Table 1) to the narrower and shorter NLA filaments.

Comparison with the $\mathrm{HCN}$ and $\mathrm{HNCO}$ data (not shown) indicate that some $\mathrm{HCO}^{+}$NLAs are also seen as absorption features in these tracers (Figure 13). A few $\mathrm{HCO}^{+}$NLA segments go into emission where their surroundings are dim in $\mathrm{HCN}$ or HNCO such as near the projected edges of the cloud. These filaments are confined to within a few arc-seconds of the cloud's edge, indicating that they may be close to the surface of G0.253+0.016.

The distribution of $\mathrm{HCO}^{+}$absorption features have no correlation with the underlying structure of G0.253+0.016 seen in other tracers. G0.253+0.016 is filamentary in optically thin emission lines such as $\mathrm{H}^{13} \mathrm{CN}$ where $\mathrm{G} 0.253+0.016$ resembles a bow shock propagating toward the east. Figure 14 


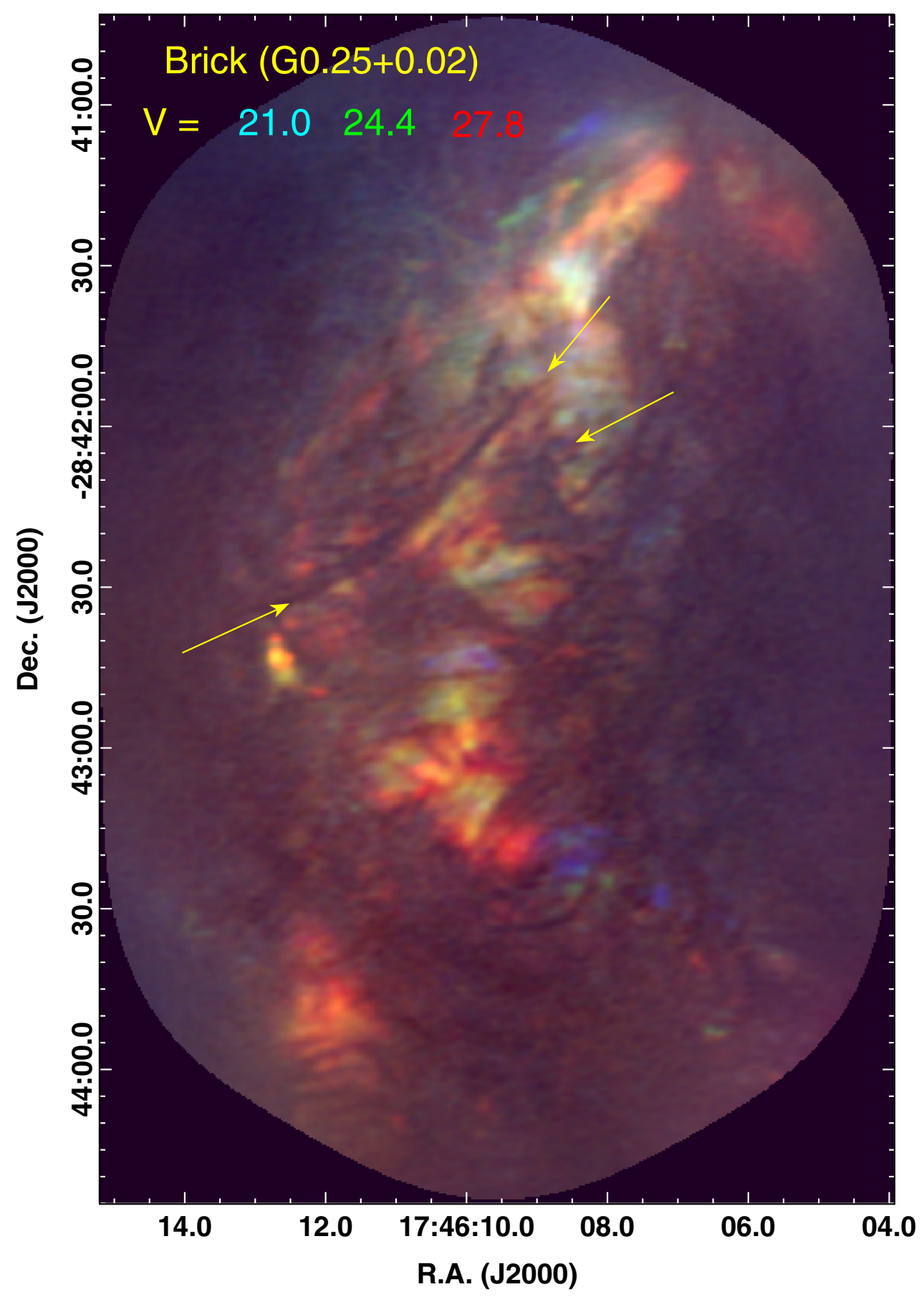

Figure 4. This figure shows $\mathrm{HCO}^{+}$channels centered at $V_{\mathrm{LSR}}=21.0$ (blue), 24.4 (green), and 27.8 (red) $\mathrm{km} \mathrm{s}^{-1}$. (A color version of this figure is available in the online journal.)

shows the optically thin $\mathrm{H}^{13} \mathrm{CO}^{+}$in three broad velocity ranges $\mathrm{V}_{\mathrm{LSR}}=10.8$ to $27.8 \mathrm{~km} \mathrm{~s}^{-1}$ (blue), $31.2-41.4 \mathrm{~km} \mathrm{~s}^{-1}$ (green), and $44.8-61.8 \mathrm{~km} \mathrm{~s}^{-1}$ (red). The east-facing bow shapes are not visible in the optically thick $\mathrm{HCO}^{+}$(or $\mathrm{HCN}$ ) data presumably because this emission is area-filling and these tracers only probe cloud structure to the $\tau \approx 1$ surface. The emission-line structure of G0.253+0.016 is discussed in Rathborne et al. (2014a). For reference, Figure 15 shows the $3 \mathrm{~mm}$ continuum emission from Rathborne et al. (2014a). It is unclear what fraction of this emission is produced by dust and what fraction is produced by free-free emission.

\subsection{Opacity and Excitation Conditions for $\mathrm{HCO}^{+}$}

The typical $\mathrm{HCO}^{+}$brightness temperature in both the ALMA and single dish observations is only about $2-4 \mathrm{~K}$ (Rathborne et al. 2014a, 2014b), considerably lower than the gas temperatures of $60-80 \mathrm{~K}$ inferred from $\mathrm{NH}_{3}$ or $\mathrm{H}_{2} \mathrm{CO}$ observations (Lis et al. 2001; Mills \& Morris 2013; Ao et al. 2013) and even lower than the dust temperature, which has been estimated to be around $19 \mathrm{~K}$ in the cloud center and about $27 \mathrm{~K}$ at the cloud surface (Lis et al. 2001; Pierce-Price et al. 2000; Longmore et al. 2012). The ALMA data shows that the $\mathrm{HCO}^{+}$emission is area 


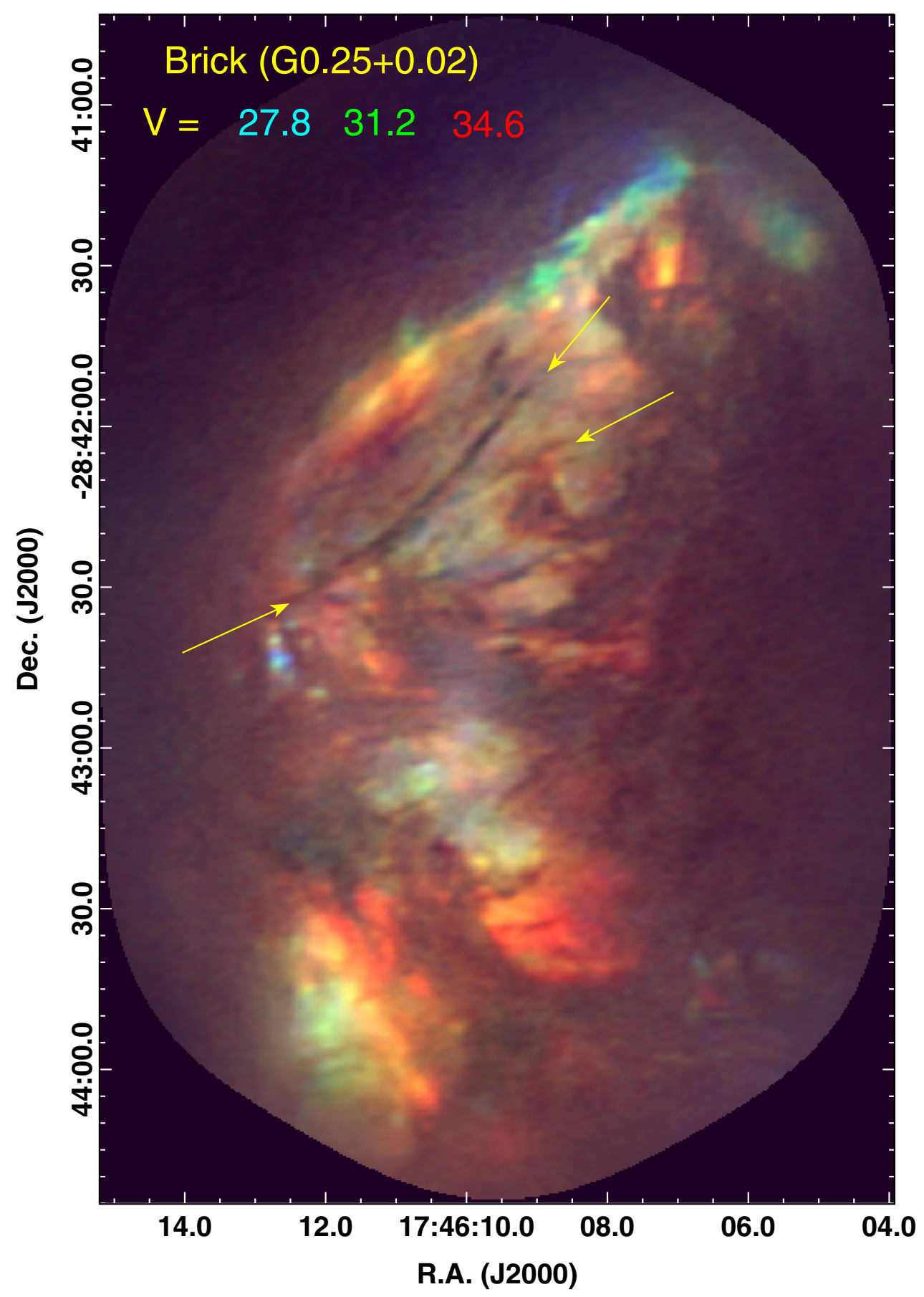

Figure 5. This figure shows $\mathrm{HCO}^{+}$channels centered at $V_{\mathrm{LSR}}=27.8$ (blue), 31.2 (green), and 34.6 (red) $\mathrm{km} \mathrm{s}^{-1}$. (A color version of this figure is available in the online journal.)

filling at arcsecond scales. Thus, the low brightness temperature must be due to low excitation temperature at the $\tau_{12}=1$ surface, implying densities much lower than the critical density for the $J=1-089 \mathrm{GHz}$ transition of $\mathrm{HCO}^{+}$(for which the critical density is $\sim 2 \times 10^{5} \mathrm{~cm}^{-3}$ ).

Comparison with the $\mathrm{H}^{13} \mathrm{CO}^{+}$ALMA images demonstrates that the $\mathrm{HCO}^{+}$emission is optically thick at most locations. The area-integrated intensity ratio of $\mathrm{HCO}^{+}$divided by the areaintegrated ratio of $\mathrm{H}^{13} \mathrm{CO}^{+}$is $R_{12 / 13}=\mathrm{I}\left(\mathrm{HCO}^{+}\right) / \mathrm{I}\left(\mathrm{H}^{13} \mathrm{CO}^{+}\right)=$ $10 \pm 1$ for the northern part of G0.253+0.016 and $R_{12 / 13}=$ $11 \pm 1$ for the southern part for integration areas ranging from
0.3 to $2.1 \operatorname{armin}^{2}$. The maximum $\mathrm{HCO}^{+}$brightness $(0.23 \mathrm{Jy}$ beam $^{-1}$ or $12.3 \mathrm{~K}$ ) occurs in a small $3^{\prime \prime}$ by $8^{\prime \prime}$ elongated knot near the southern part of G0.253+0.016 at J2000 = 17:46:08.5, $-28: 43: 33$ at $V_{\mathrm{LSR}}=55 \mathrm{~km} \mathrm{~s}^{-1}$. At this location, the ratio $R_{12 / 13} \approx 12$. Thus, the observed brightness ratio, $R_{12 / 13}$ is lower than the abundance ratio, $X_{12 / 13}=\left[{ }^{12} C\right] /\left[{ }^{13} C\right]$.

Langer \& Penzias (1990) measured the abundance ratio $X_{12 / 13}$ as a function of Galactocentric radius finding that in the CMZ $X_{12 / 13} \approx 24$. In a more recent study, Riquelme et al. (2010) found a similar ratio in most parts of the CMZ. However, near cloud edges, some ratios as high as 40 were found. 


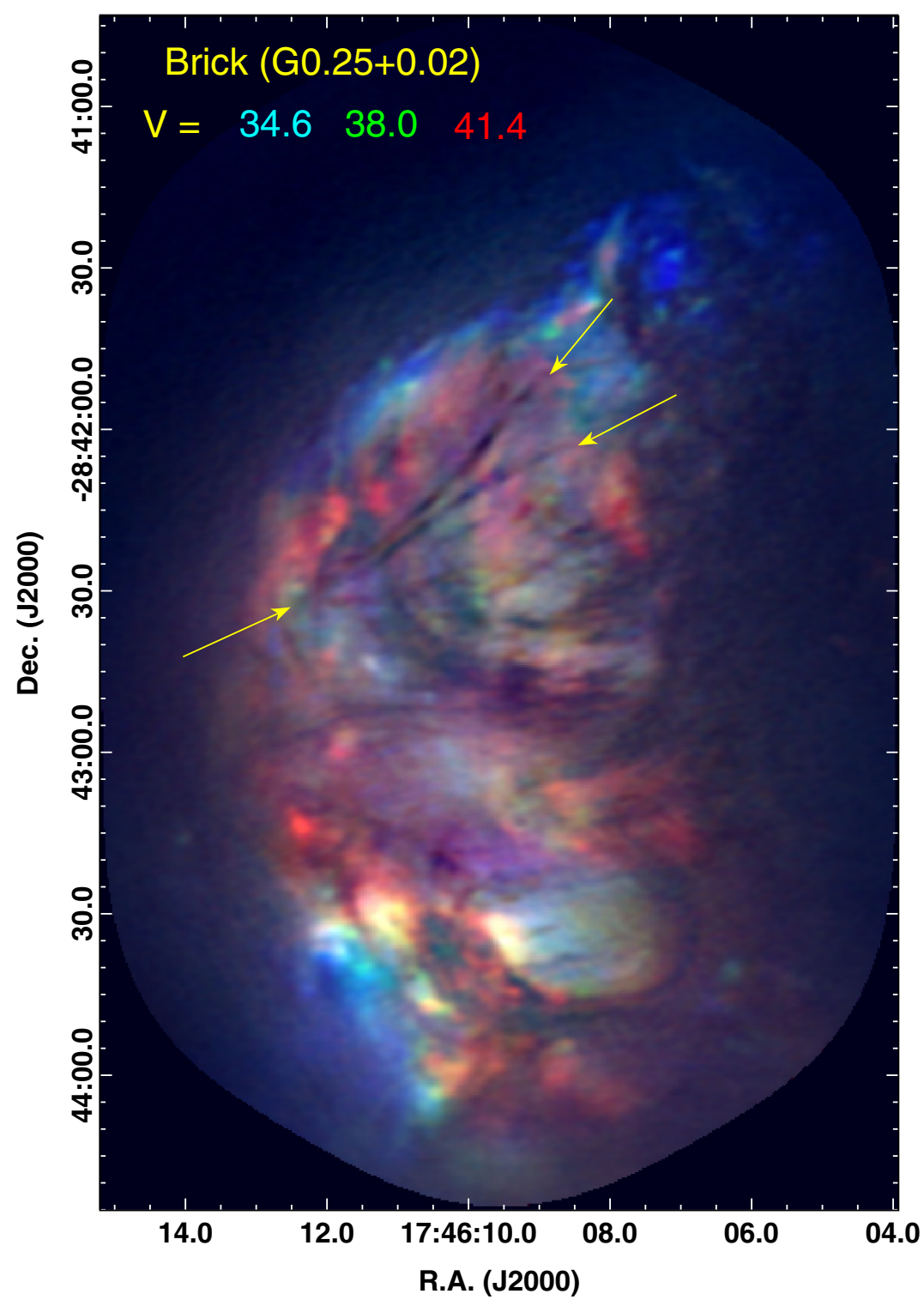

Figure 6. This figure shows $\mathrm{HCO}^{+}$channels centered at $V_{\mathrm{LSR}}=34.6$ (blue), 38.0 (green), and 41.4(red) $\mathrm{km} \mathrm{s}^{-1}$.

(A color version of this figure is available in the online journal.)

Table 1

$\mathrm{HCO}^{+}$-selected Filaments in Absorption Toward the Brick

\begin{tabular}{|c|c|c|c|}
\hline $\begin{array}{l}\alpha \\
\mathrm{J} 2000\end{array}$ & $\begin{array}{c}\delta \\
\mathrm{J} 2000\end{array}$ & $\begin{array}{c}V_{\mathrm{LSR}} \\
\left(\mathrm{km} \mathrm{s}^{-1}\right)\end{array}$ & Comments \\
\hline $17: 46: 09.2$ & $-28: 41: 54$ & 21 to 45 & BLA 1 northwest end \\
\hline $17: 46: 10.6$ & $-28: 42: 14$ & 21 to 45 & BLA1 core \\
\hline $17: 46: 11.8$ & $-28: 42: 25$ & 21 to 45 & BLA 1 southeast end \\
\hline $17: 46: 08.9$ & $-28: 42: 05$ & 21 to 45 & BLA2 west end \\
\hline $17: 46: 09.9$ & $-28: 42: 12$ & 34 to 45 & BLA2 core 1 \\
\hline $17: 46: 11.1$ & $-28: 42: 22$ & 21 to 45 & BLA 2 core 2 \\
\hline $17: 46: 12.9$ & $-28: 42: 34$ & 18 to 45 & BLA2 east end \\
\hline $17: 46: 10.6$ & $-28: 41: 55$ & 11 to 21 & $6^{\prime \prime}$ radius loop \\
\hline 17:46:10.6 & $-28: 41: 55$ & 11 to 21 & $13^{\prime \prime}$ radius loop \\
\hline $17: 46: 10.3$ & $-28: 42: 28$ & 31 to 38 & NLA 1 west end \\
\hline $17: 46: 11.9$ & $-28: 42: 33$ & 31 to 38 & NLA 1 east end \\
\hline $17: 46: 07.5$ & $-28: 42: 50$ & 21 to 45 & NLA 2 cluster; west end \\
\hline $17: 46: 11.7$ & $-28: 42: 50$ & 21 to 45 & NLA 2 cluster; east end \\
\hline $17: 46: 06.6$ & $-28: 43: 46$ & 31 to 48 & NLA 3 cluster; west end \\
\hline $17: 46: 10.6$ & $-28: 43: 30$ & 31 to 48 & NLA 3 cluster; east end \\
\hline
\end{tabular}

Milam et al. (2005) found a lower value in the $\mathrm{CMZ}$ of $X_{12 / 13} \approx$ $17 \pm 7$. Thus, there may a some intrinsic variation possibly do to local enrichments or depletion such as might be caused by local stellar processing and injection into the interstellar medium (ISM) or isotope-selective photo-dissociation (Röllig \& Ossenkopf 2013; Lyons \& Young 2005; Bally \& Langer 1982).

Assuming that the $X_{12 / 13}$ is 24 in $\mathrm{G} 0.253+0.016$, that the excitation temperatures of $\mathrm{H}^{13} \mathrm{CO}^{+}$and $\mathrm{HCO}^{+}$are the same, and that for $\mathrm{HCO}^{+}$the observed brightness is equal to the excitation temperature, as expected for an optically thick emission line, the $R_{12 / 13}$ ratio of $\sim 10$ indicates that the mean emission line optical depth of $\mathrm{HCO}^{+}$is

$$
\tau_{12} \approx X_{12 / 13} \ln \left[R_{12 / 13} /\left(R_{12 / 13}-1\right)\right] \sim 2.5 .
$$

For $X_{12 / 13}$ ranging from 17 to $40, \tau_{12}, R_{12 / 13}=10$ implies that $\tau_{12}$ ranges from 1.5 to 2.5 .

In the $\mathrm{HCO}^{+}$photosphere (where $\tau_{12} \sim 1$ ), the density must be much lower than the critical density, implying sub-thermal 


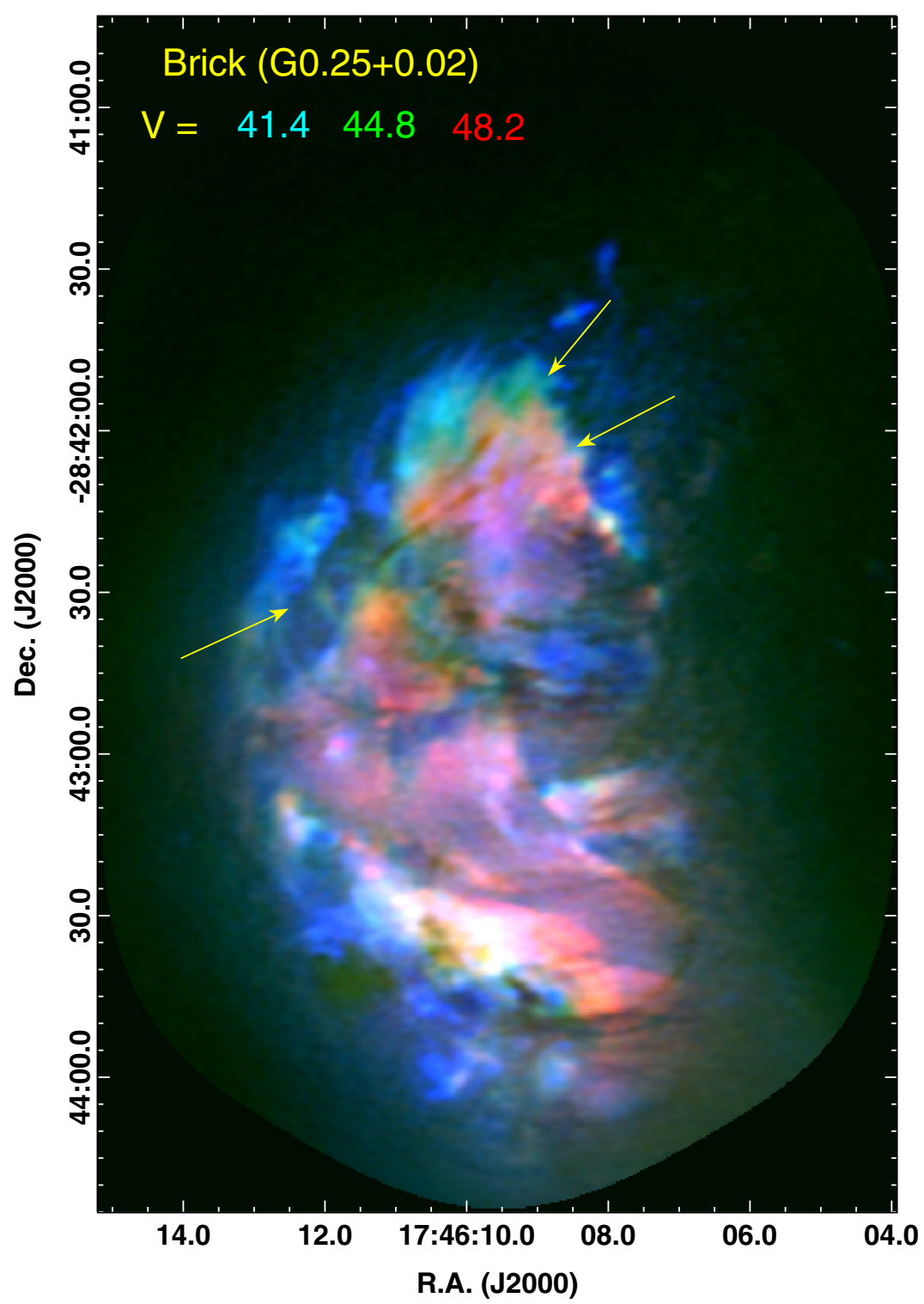

Figure 7. This figure shows $\mathrm{HCO}^{+}$channels centered at $V_{\mathrm{LSR}}=41.4$ (blue), 44.8 (green), and 48.2 (red) $\mathrm{km} \mathrm{s}^{-1}$.

(A color version of this figure is available in the online journal.)

excitation, and densities much lower than the mean density of $\mathrm{G} 0.253+0.016$. The brightness temperature of $\mathrm{HCO}^{+}$implies that $T_{e x}<10 \mathrm{~K}$ over most of the $\mathrm{G} 0.253+0.016$ photosphere. Clark et al. (2013) modeled both the gas and dust temperatures in G0.253+0.016, finding that high gas temperatures and lower dust temperatures can be explained by a very high cosmic-ray flux and ionization rate. The enhanced cosmic-ray flux may also enhance the abundances of certain molecules such as methanol (Yusef-Zadeh et al. 2013) and $\mathrm{HCO}^{+}$.

$\mathrm{HCO}^{+}$is the most abundant molecular ion next to $\mathrm{H}_{3}^{+}$in the molecular phase of the ISM. Collisional excitation of its $J=1-0$ transition requires a collision partner density $\left(\mathrm{H}_{2}\right.$ and $\left.\mathrm{He}\right)$ of $n>$ $10^{4} \mathrm{~cm}^{-3}$ to thermalize. $\mathrm{HCO}^{+}$has as relatively high abundance of $n\left(\mathrm{HCO}^{+}\right) / n\left(\mathrm{H}_{2}\right) \sim 10^{-9}$ to $10^{-7}$ in nearby molecular clouds. $\mathrm{HCO}^{+}$is created by the reaction $\mathrm{CO}+\mathrm{H}_{3}^{+}->\mathrm{HCO}^{+}+\mathrm{H}_{2}$ and destroyed by reactions with $\mathrm{H}_{2}, \mathrm{HCO}^{+}+\mathrm{H}_{2}->\mathrm{H}_{2} \mathrm{CO}+\mathrm{H}^{+}$ or by recombination with electrons, $\mathrm{HCO}^{+}+\mathrm{e}^{-}->\mathrm{CO}+\mathrm{H}^{+}$. The large abundance of $\mathrm{H}_{3}^{+}$in the CMZ (Goto et al. 2008, 2011) may drive the $\mathrm{HCO}^{+}$abundance in the $\mathrm{CMZ}$ to values near the upper-end of the above range.

The column densities of the absorption filaments can be estimated from the absorption optical depth. Following Liszt \& Lucas (2004) the column density of a linear molecule having dipole moment $\mu$ (about 4 Debye for $\mathrm{HCO}^{+}$) absorbing from its ground state is

$$
N_{0}=\frac{8.0 \times 10^{12} \int \tau_{10} d V}{\mu^{2}\left(1-\exp \left(-h v_{10} / k T_{e x}\right)\right.},
$$

where $v_{10}$ is the $J=10-0$ frequency $(89.1885 \mathrm{GHz})$ for $\mathrm{HCO}^{+}$ and $T_{e x}$ is the excitation temperature. Measuring the $\mathrm{HCO}^{+}$ fluxed in the filament and comparing this with the surrounding off-filament pixels indicates that typically about one-half of the background light is absorbed, implying a typical absorption optical depth of about 0.7. For the broad-line filaments, $d V \sim$ $10-30 \mathrm{~km} \mathrm{~s}^{-1}$ and $\tau_{10} \sim 0.2-2$ with a mean value of 0.7 . For a typical equivalent width (integral of absorption optical depth 


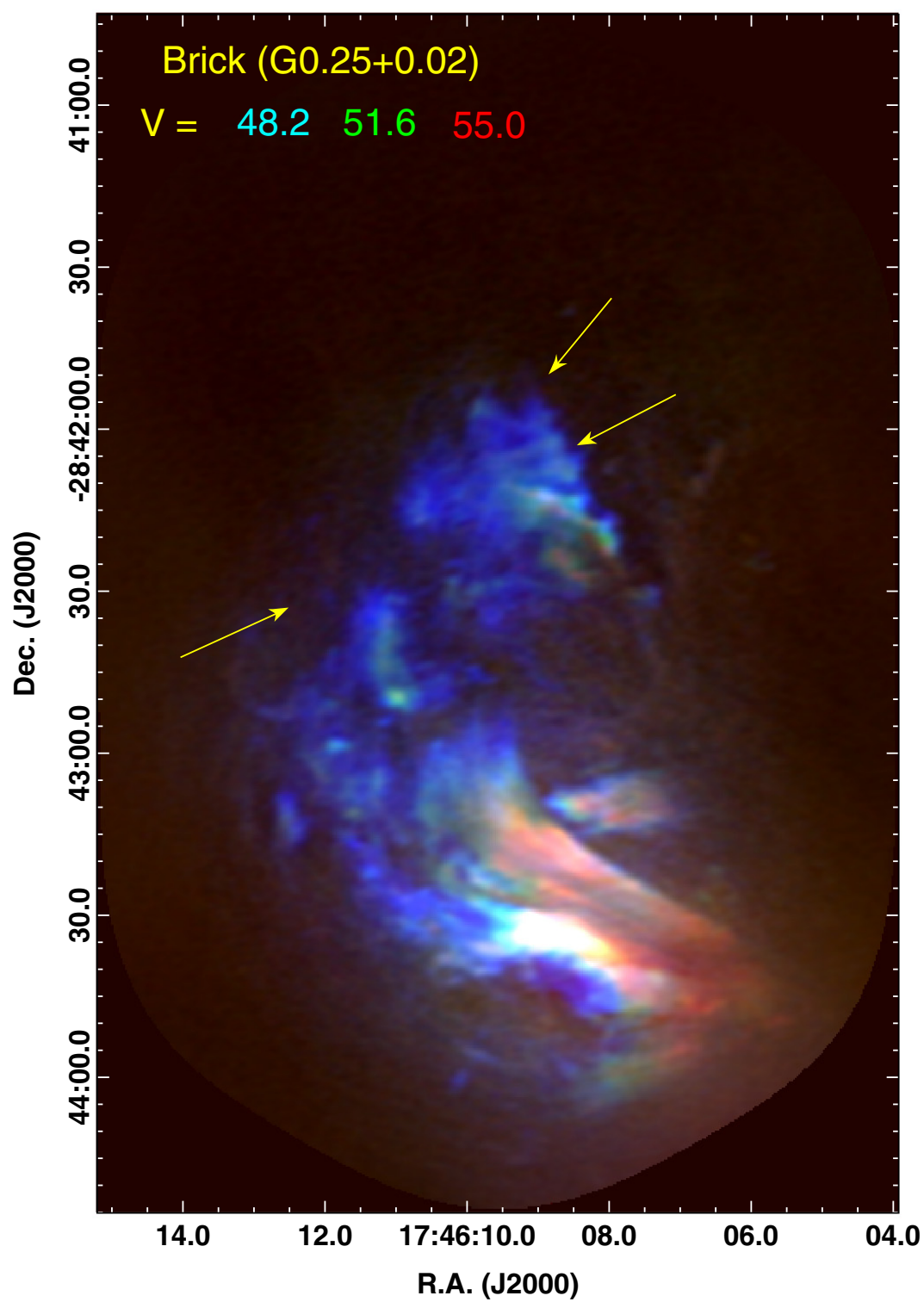

Figure 8. This figure shows $\mathrm{HCO}^{+}$channels centered at $V_{\mathrm{LSR}}=48.2$ (blue), 51.6 (green), and 55.0 (red) $\mathrm{km} \mathrm{s}^{-1}$.

(A color version of this figure is available in the online journal.)

over the line width) of $10 \mathrm{~km} \mathrm{~s}^{-1}$, the column density of $\mathrm{HCO}^{+}$ absorbers is $N_{0} \approx 10^{13} \mathrm{~cm}^{-2}$ for $T_{\mathrm{ex}}=2.73 \mathrm{~K}$, and $6 \times 10^{13} \mathrm{~cm}^{-2}$ for $T_{\mathrm{ex}}=10 \mathrm{~K}$.

Table 2 lists the excitation temperatures $\left(T_{e x}\right)$, opacities $(\tau)$, and brightness temperatures $\left(T_{R}\right)$ for the $J=1-0$ transition of $\mathrm{HCO}^{+}$for a range of molecular hydrogen volume densities $(n)$ and $\mathrm{HCO}^{+}$column densities $(N)$ using the radiative transfer code RADEX (van der Tak et al. 2007). These calculations show that an $\mathrm{H}_{2}$ density of $1 \times 10^{3} \mathrm{~cm}^{-3}$ an $\mathrm{HCO}^{+}$column density of $1-10 \times 10^{13} \mathrm{~cm}^{-2}$ and line width $\Delta V=10 \mathrm{~km} \mathrm{~s}^{-1}$ in a gas with a kinetic temperature of $60 \mathrm{~K}$ results in an excitation temperature of about $2.8-3.2 \mathrm{~K}$, an optical depth $0.8-7$ in the $J=1-0$ line. These parameters would produce the absorption seen in the NLA filaments. The BLA filaments require a slightly lower $\mathrm{H}_{2}$ density to produce the low observed brightness temperature and an opacity of the order of unity required to explain the amount of background attenuation. The $\mathrm{HCO}^{+}$radiation field of $\mathrm{G} 0.253+0.016$ may contribute to the excitation of the upper state by effectively raising the background radiation temperature (radiative pumping). Detailed modeling of the excitation conditions and radiative transfer using realistic cloud geometries is needed to determine if the low observed brightness temperature of the NLA and BLA filaments can be used to place a lower bound on separation between the absorbing gas and G0.253+0.016.

The width of these filaments is less than about $2^{\prime \prime}$ or about $0.08 \mathrm{pc}$. (If only $1^{\prime \prime}$ wide, the filaments must be completely dark and only a lower bound can be placed on their optical depth. Higher angular resolution ALMA observations will be obtained during ALMA Cycle 1 to measure their actual widths.) From the estimated column density and $\mathrm{HCO}^{+}$abundance $X\left(\mathrm{HCO}^{+}\right)=$ $10^{-7}$, assuming that the depth of the filament is comparable to its projected width, the higher excitation temperature $\left(T_{e x}=\right.$ $10 \mathrm{~K}$ ) would imply an $\mathrm{H}_{2}$ column density of $6 \times 10^{20} \mathrm{~cm}^{-2}$ and a volume density of around 2.5 to $5 \times 10^{3} \mathrm{~cm}^{-3}$. A density larger than this value would drive the absorption filaments 


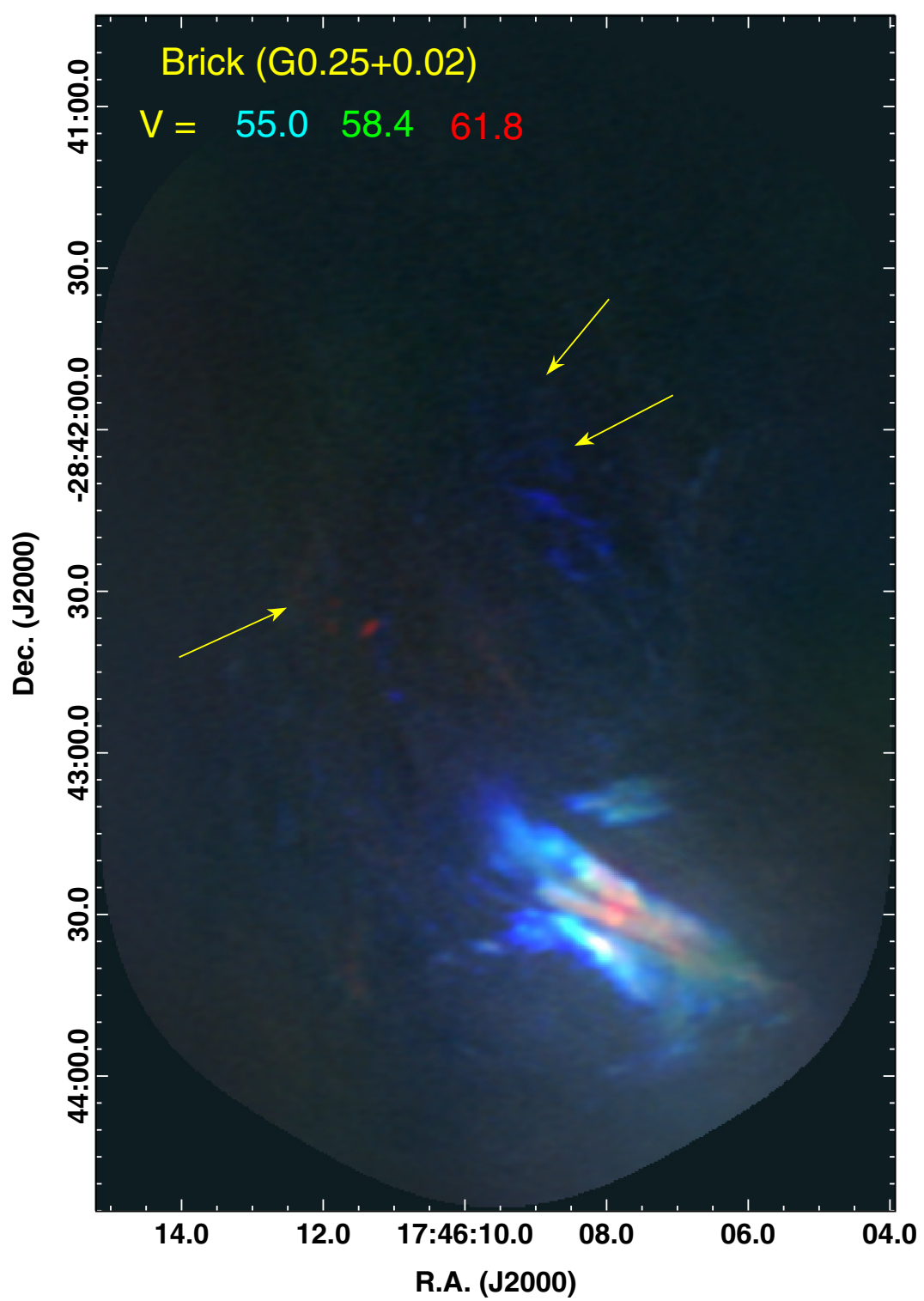

Figure 9. This figure shows $\mathrm{HCO}^{+}$channels centered at $V_{\mathrm{LSR}}=55.0$ (blue), 58.4 (green), and 61.8(red) $\mathrm{km} \mathrm{s}^{-1}$. (A color version of this figure is available in the online journal.)

Table 2

RADEX ${ }^{1}$ Calculations for the $J=1-0 \mathrm{HCO}^{+}$Transition

\begin{tabular}{|c|c|c|c|c|c|c|c|c|c|}
\hline \multirow{2}{*}{$\begin{array}{l}\log n\left(\mathrm{H}_{2}\right) \\
\log N\left(\mathrm{HCO}^{+}\right)\end{array}$} & \multicolumn{3}{|c|}{$T_{\mathrm{ex}}$} & \multicolumn{3}{|c|}{$\tau$} & \multicolumn{3}{|c|}{$T_{R}$} \\
\hline & 13 & 14 & 15 & 13 & 14 & 15 & 13 & 14 & 15 \\
\hline 2.000 & 2.74 & 2.78 & 3.15 & 0.85 & 8.41 & 72.9 & 0.005 & 0.004 & 0.355 \\
\hline 2.477 & 2.76 & 2.87 & 3.78 & 0.84 & 8.10 & 58.1 & 0.016 & 0.118 & 0.909 \\
\hline 3.000 & 2.84 & 3.17 & 5.23 & 0.82 & 7.24 & 37.9 & 0.053 & 0.366 & 2.254 \\
\hline 3.477 & 3.07 & 3.83 & 7.69 & 0.75 & 5.74 & 21.5 & 0.149 & 0.950 & 4.624 \\
\hline 4.000 & 3.84 & 5.49 & 12.4 & 0.57 & 3.55 & 10.1 & 0.415 & 2.426 & 9.275 \\
\hline 4.477 & 6.30 & 9.33 & 20.1 & 0.30 & 1.69 & 4.60 & 0.849 & 5.078 & 16.77 \\
\hline 5.000 & 38.7 & 37.4 & 41.4 & 0.03 & 0.29 & 1.57 & 1.210 & 8.670 & 30.16 \\
\hline 5.477 & -19 & -34 & 152 & -0.04 & -0.2 & 0.31 & 1.002 & 8.681 & 39.68 \\
\hline
\end{tabular}

Notes. [1]: The tabulated values assume a background radiation temperature of $2.73 \mathrm{~K}$, a gas kinetic temperature of $60 \mathrm{~K}$, and a line width of $10 \mathrm{~km} \mathrm{~s}^{-1}$. Based on the online RADEX tool available at http://www.sron.rug.nl/ vdtak/radex/radex.php. 

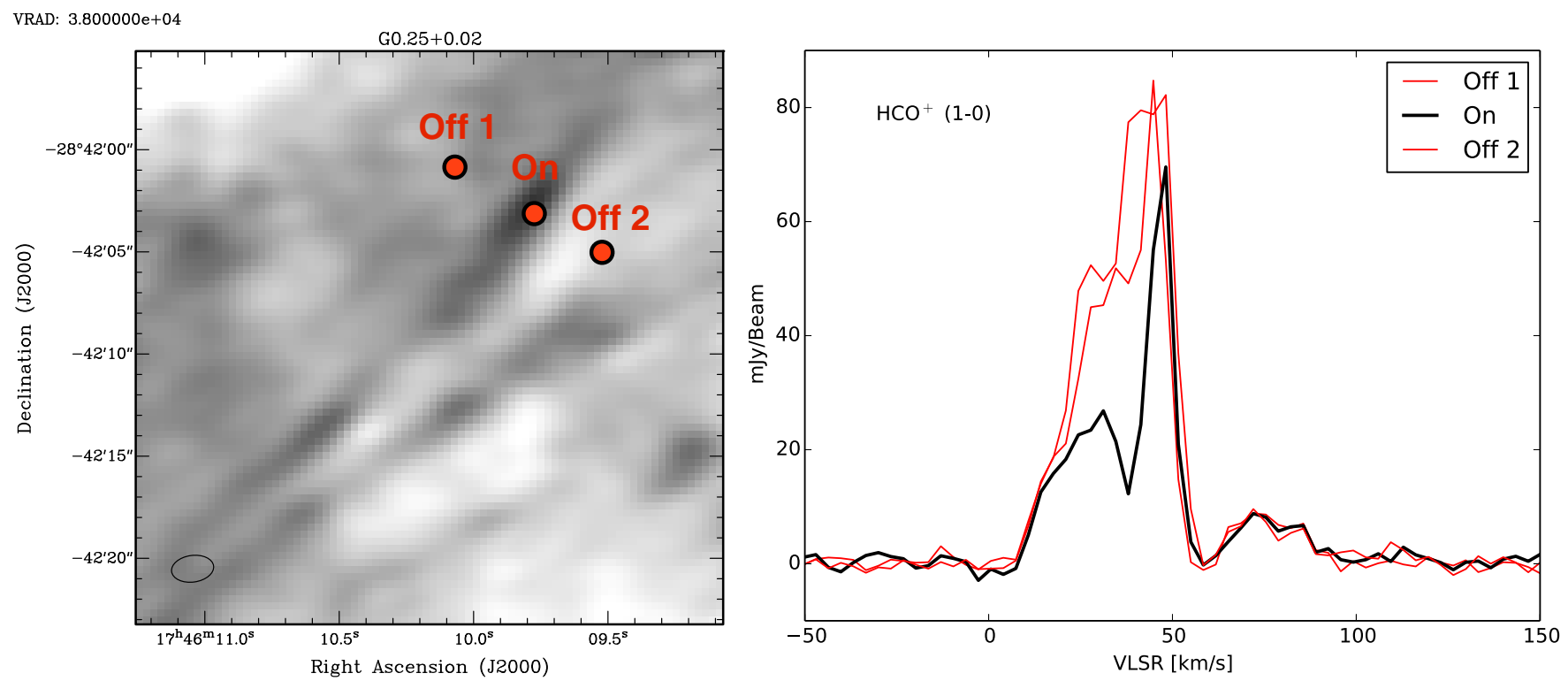

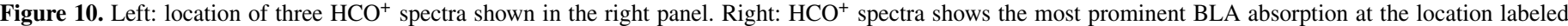
"On" (thick black line) and two off positions (thin red lines).

(A color version of this figure is available in the online journal.)
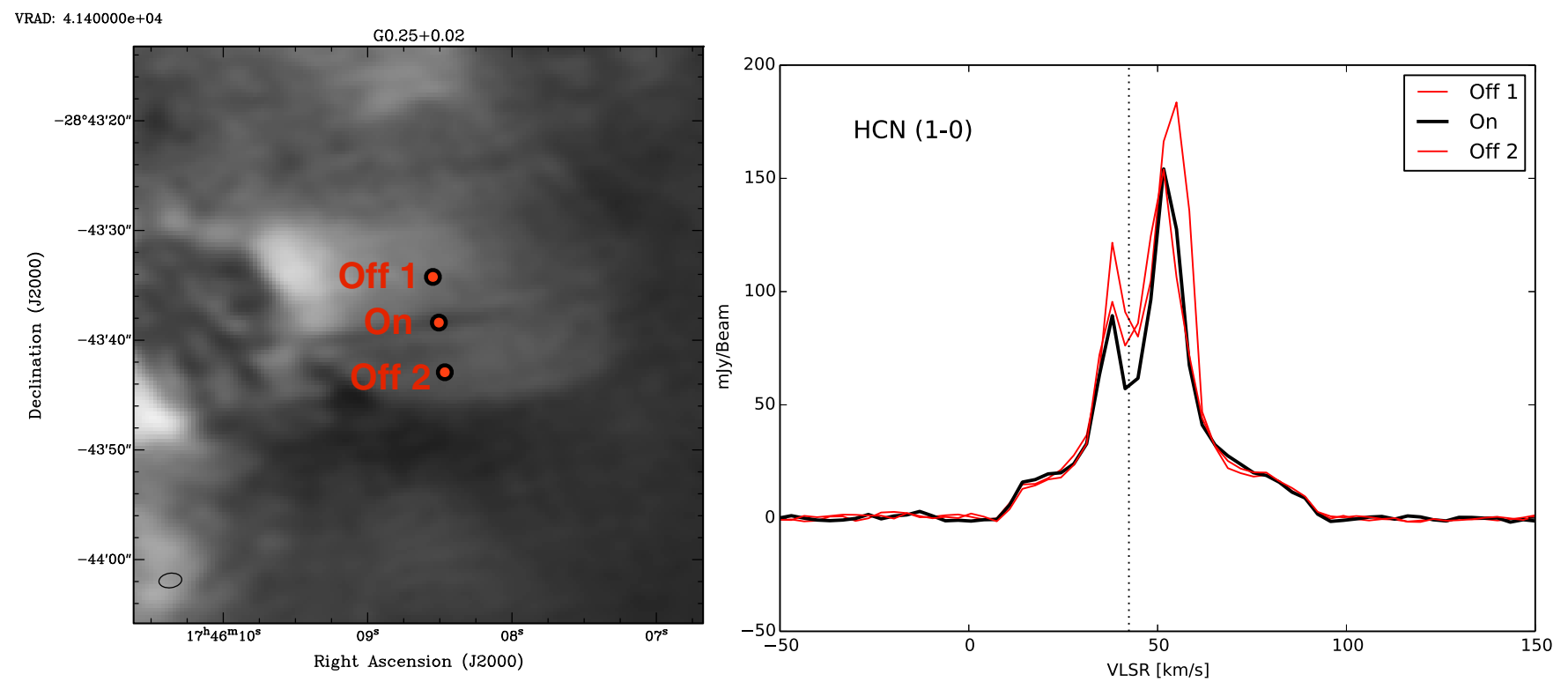

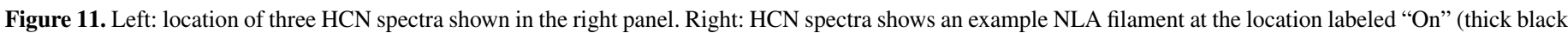
line) and two off positions (thin red lines). The dotted vertical line shows the central velocity of the absorption feature.

(A color version of this figure is available in the online journal.)

into emission in $\mathrm{HCO}^{+}$. A lower $\mathrm{HCO}^{+}$abundance increases the estimated volume density proportionally and conversely, a higher $\mathrm{HCO}^{+}$abundance decreases the $\mathrm{H}_{2}$ volume density. Thus, either the $\mathrm{HCO}^{+}$abundance must be higher than $X\left(\mathrm{HCO}^{+}\right)=$ $10^{-7}$, or the line-of-sight depth of the filaments must be larger than their projected widths.

It is possible that the filaments are sheets seen edge-on so that the line-of-sight depth is much larger than projected width of the filament. In such a sheet, the column density to volume density ratio can be much larger than for a cylinder. For a given $\mathrm{HCO}^{+}$abundance, the observed column density requires a volume density that is lower by the ratio of the line-of-sight depth of the sheet divided by its projected width on the plane of the sky. Such edge-on sheets may be produced by shocks propagating into molecular media at right angles to our line of sight. With $n\left(\mathrm{H}_{2}\right)<5 \times 10^{3} \mathrm{~cm}^{-3}$, the densities in the absorption filaments must be lower than the density at the $\tau \sim 1$ surface in $\mathrm{HCO}^{+}$which is sub-thermally excited, and well below the mean density of $\mathrm{G} 0.253+0.016, n\left(\mathrm{H}_{2}\right) \approx 7 \times 10^{4} \mathrm{~cm}^{-3}(\mathrm{Lis}$ et al. 2001; Longmore et al. 2012; Rathborne et al. 2014b).

Inspection of the $\mathrm{HCO}^{+}$and $\mathrm{HCN}$ data show that both tracers are heavily absorbed on the blueshifted, approaching side of the spectral line profiles. Thus, there is a pervasive layer of subthermally excited, low-density $\mathrm{HCO}^{+}$and $\mathrm{HCN}$ bearing gas in front of $\mathrm{G} 0.253+0.016$. If associated with $\mathrm{G} 0.253+0.016$, this gas would indicate the presence of an extended layer expanding from $\mathrm{G} 0.253+0.016$ at velocities $10-20 \mathrm{~km} \mathrm{~s}^{-1}$ over the entire face of this cloud. 


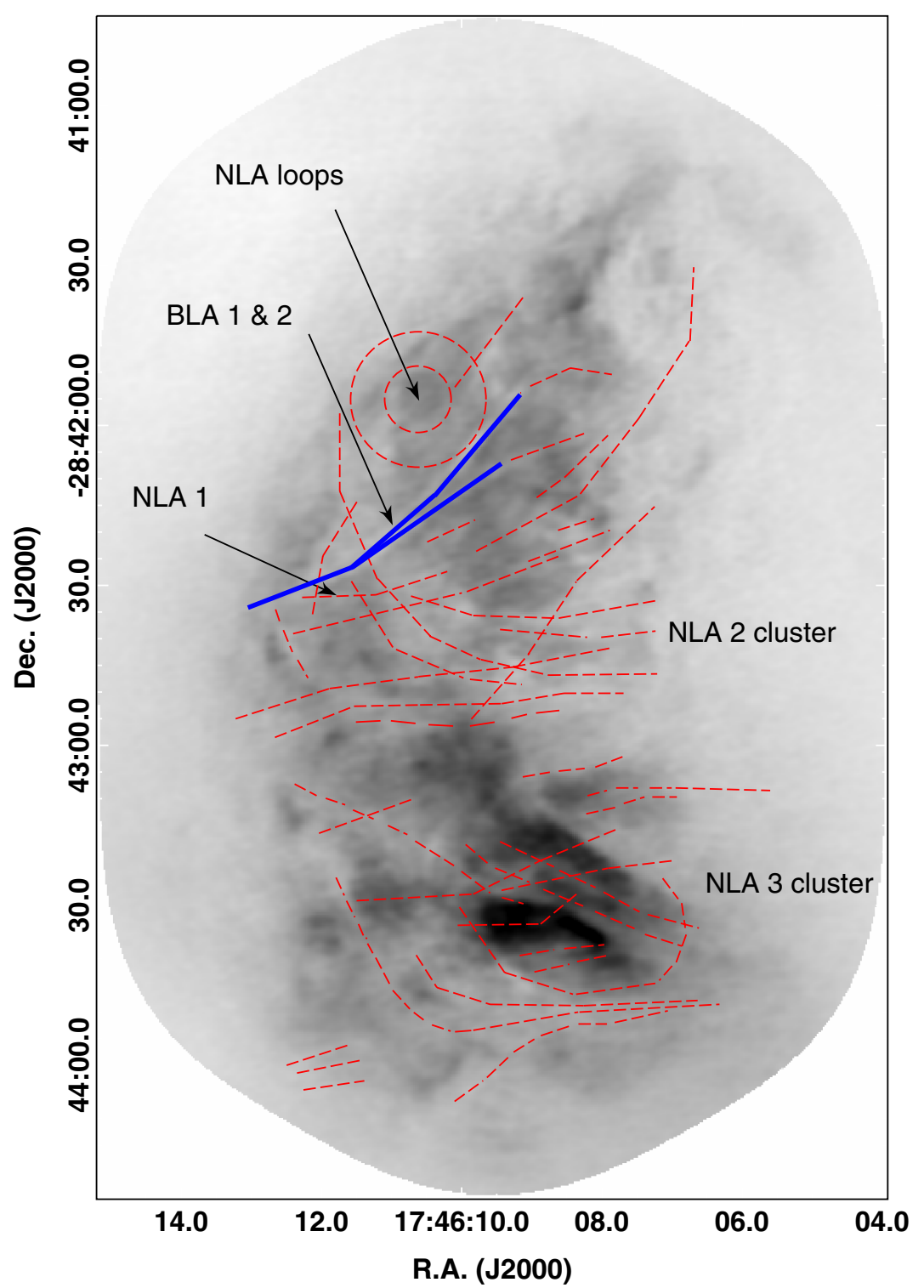

Figure 12. Image shown in Figure 1 with the locations of filaments superimposed. Thick solid lines show BLA filaments; thin dashed lines indicate locations of some of the NLA filaments.

(A color version of this figure is available in the online journal.)

While NLA filaments near $V_{\mathrm{LSR}}=0 \mathrm{~km} \mathrm{~s}^{-1}$ could be located anywhere between the Sun and G0.253+0.016, the higher radial velocity NLA filaments with $V_{\mathrm{LSR}}>10 \mathrm{~km} \mathrm{~s}^{-1}$ are most likely to be in the CMZ (local gas is found between $V_{\mathrm{LSR}} \sim-10$ to $+10 \mathrm{~km} \mathrm{~s}^{-1}$ and the Galactic spiral arms between the Sun and the $\mathrm{CMZ}$ are at $V_{\mathrm{LSR}} \sim-10$ to $-65 \mathrm{~km} \mathrm{~s}^{-1}$ ). Near the projected edge of G0.253+0.016 some NLA filaments go faintly into emission in $\mathrm{HCN}$ and $\mathrm{HNCO}$. Because these features disappear a few arc seconds beyond the cloud's edge, they must be physically close to G0.253+0.016, suggesting that they trace sub-thermal $\mathrm{HCO}^{+}$and $\mathrm{HCN}$ molecules on the near surface of the cloud.

\section{THE DYNAMIC STATE OF G0.253+0.016: COLLAPSE, EXPANSION, OR BOTH?}

Rathborne et al. (2014b) analyzed millimeter and submillimeter-wave emission in multiple transitions from G0.253+0.016 obtained with the Mopra and APEX single-dish telescopes. In addition to the large north-south velocity gradient, they found that the optically thick dense-gas tracers were systematically redshifted with respect to the optically thin and hot gas tracers, indicating radial motions which could be interpreted as either due to expansion or collapse. If the brighter, redshifted emission in optically thick species such as $\mathrm{HCO}^{+}$and $\mathrm{HCN}$ originates in an externally heated layer at the front surface, then the cloud is contracting. Rathborne et al. (2014b) refer to this as their "Baked Alaska" model. The bright blueshifted emission expected from the far-side heated layer would be absorbed by $\mathrm{HCO}^{+}$molecules in the supersonically turbulent, colder, cloud interior. This interpretation requires that the excitation temperature of the $\mathrm{HCO}^{+} J=1-0$ transition in the cloud surface layer producing the redshifted line component must be higher than excitation temperature of the component producing the lowervelocity and lower brightness-temperature emission in the cloud interior. Alternatively, if the excitation temperature in the layer where the optical depths reach a value of the order of unity is low compared to the excitation temperature deep in the cloud 


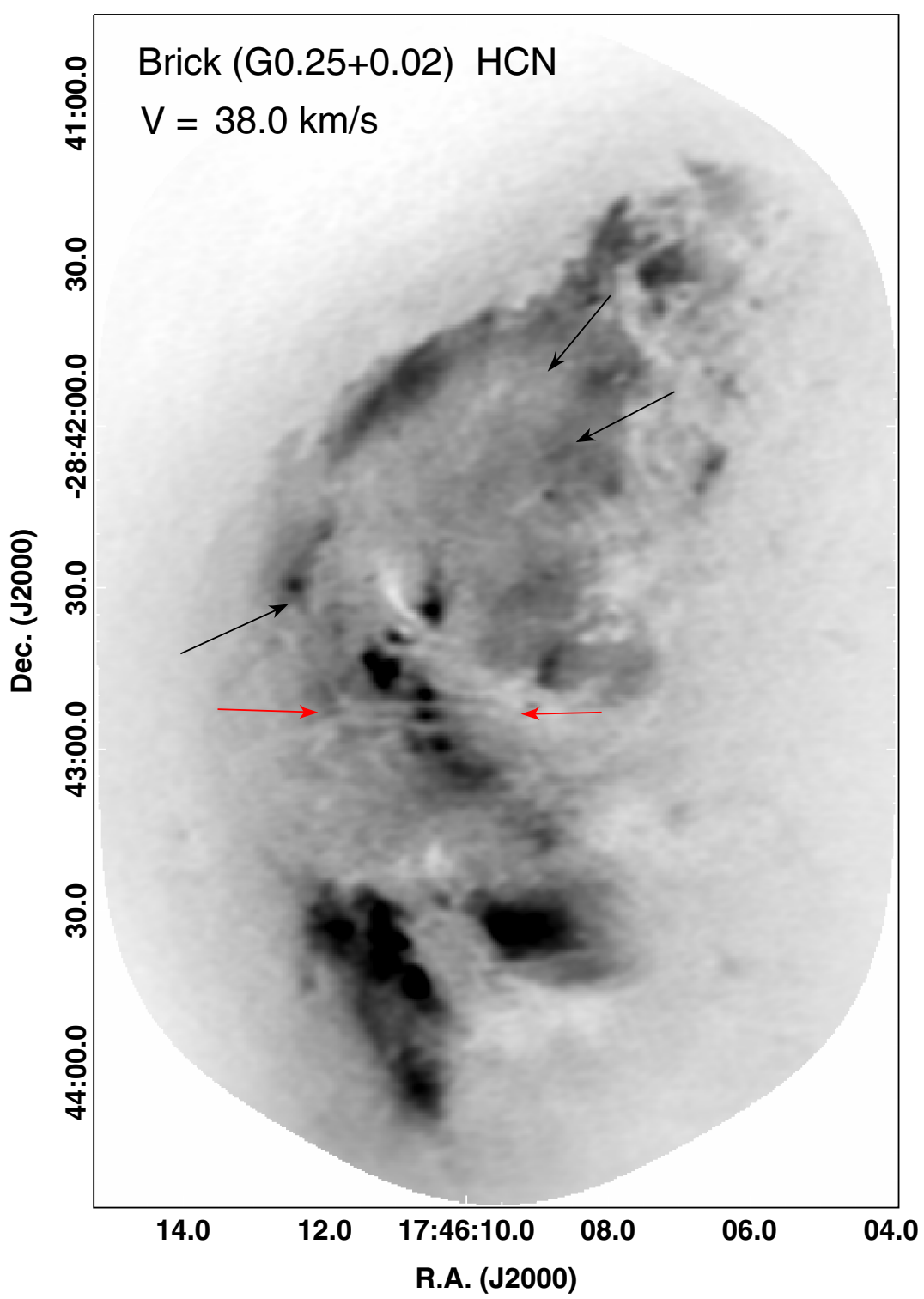

Figure 13. $\mathrm{HCN}$ image at $\mathrm{V}_{\mathrm{LSR}}=38.0 \mathrm{~km} \mathrm{~s}^{-1}$ showing narrow-line absorption (NLA) filaments. The prominent broad-line absorption (BLA) filaments seen in HCO ${ }^{+}$ are not seen in this tracer. The two horizontal arrows mark the locations of several NLA filaments in the NLA 2 cluster which also absorb in HCN.

(A color version of this figure is available in the online journal.)

interior, then these observations can be interpreted as evidence for expansion of the cloud surface layers. For sub-thermal excitation of molecules at the cloud surface, collapse would produce redshifted absorption while expansion would produce blueshifted absorption.

At lower, blueshifted radial velocities, the $\mathrm{HCO}^{+} J=1-0$ transition is seen as a network of absorption filaments against brighter background emission. The brighter, background $\mathrm{HCO}^{+}$ emission appears to fill the synthesized ALMA beam and to cover most of the projected surface area of G0.253+0.016 with a peak brightness temperature of the order of 2 to at most $10 \mathrm{~K}$, well below the gas kinetic temperature, $T_{K}>60 \mathrm{~K}$. A peak line temperature lower than $T_{K}$ requires that either the emitting region fills only a small portion of the telescope beam ("beam dilution"), or a small fraction of the spectral channel ("frequency dilution"), or that the excitation temperature $T_{\mathrm{ex}}$ at the $\tau \approx 1$ surface be much lower than $T_{K}$. The bright $\mathrm{HCO}^{+}$ emission between the absorption filaments appears smooth on scales larger than the 1".7 ALMA beam, and continuous from one velocity channel to the next, indicating that beam and frequency dilution of the $\mathrm{HCO}^{+}$emission is unlikely. Thus, the relatively low brightness temperature of $\mathrm{HCO}^{+}$is most likely due to low excitation temperature, implying that the $\mathrm{H}_{2}$ density near the $\tau=1$ surface is much lower than the critical density for the $\mathrm{HCO}^{+} J=1-0$ transition.

The $\mathrm{HCO}^{+}$filaments are seen in absorption against this background emission, they have an even lower excitation temperature than the regions seen in emission. If these blueshifted features are at the cloud surface, the G0.253+0.016 surface layers must be expanding away from the cloud center of mass despite the large mass and gravity field. This is consistent with the second ("P-Cygni") interpretation of the single-dish data presented by Rathborne et al. (2014b).

The mass-loss rate is difficult to estimate because the density on the absorbing layer is an upper bound, the column density is a lower bound (based on an assumed $\mathrm{HCO}^{+}$abundance), 


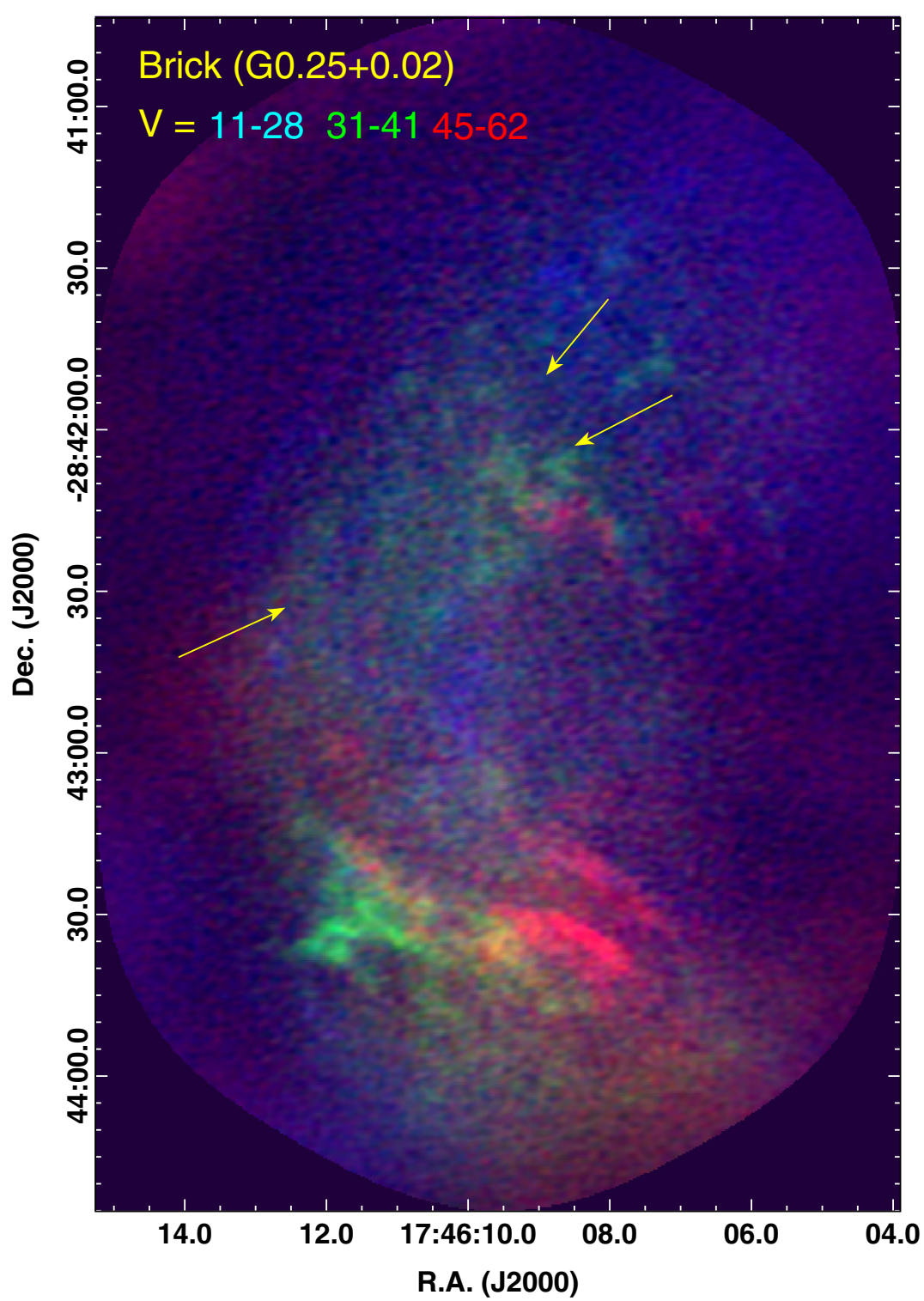

Figure 14. $\mathrm{H}^{13} \mathrm{CO}^{+}$RGB image showing the bow shape of the Brick in an optically thin tracer. The velocity ranges (in $\mathrm{km} \mathrm{s}^{-1}$ ) for the blue, green, and red are indicated in the figure.

(A color version of this figure is available in the online journal.)

and the thickness of the absorbing layer is not known. For a given density $n\left(\mathrm{H}_{2}\right)$, and assuming that all sides of the cloud are expanding in a manner similar to that side facing the Sun, the mass-loss rate is given by $\dot{M} \sim 4 \pi f R_{\text {eff }}^{2} \mu m_{\mathrm{H}} n\left(\mathrm{H}_{2}\right) V$ where $f$ is the fraction of the cloud surface area occupied by expanding gas, $R_{\text {eff }} \approx 2.7 \mathrm{pc}$ is the mean cloud radius, $n\left(\mathrm{H}_{2}\right)$ is the area averaged mean density of the NLA filaments at the cloud's surface, and $V \sim 10-20 \mathrm{~km} \mathrm{~s}^{-1}$ is the expansion speed of the absorbing layer. For $f=0.5$ and $n\left(\mathrm{H}_{2}\right)=10^{3} \mathrm{~cm}^{-3}$, set by the constraint that $\mathrm{HCO}^{+}$in the absorbing gas is severely sub-thermally excited, $\dot{M} \sim 0.03-0.06 M_{\odot} \mathrm{yr}^{-1}$. In the last $0.25 \mathrm{Myr}$, since its close passage to Sgr A, G0.253+0.016 could have lost $10 \%-20 \%$ of its mass.

It is possible that $\mathrm{G} 0.253+0.016$ is simultaneously undergoing both collapse and expansion. The presence of a maser and at least one massive clump surrounding it suggests that at least one moderate or high-mass star is forming in the cloud interior. The cold and dense cloud interior may thus be experiencing gravitational collapse and star formation. However, the lack of internal heating and absence of any other signposts of young stars other than the maser source, suggests that the cloud is only in the earliest phases of star formation. Blueshifted absorption in optically thick species such as $\mathrm{HCO}^{+}$over much of the cloud surface area is an indication that outer layers may be expanding.

\section{DISCUSSION}

The filamentary structure of molecular clouds has been recognized for decades (Bally et al. 1987a). However, the extensive arcsecond scale $\mathrm{HCO}^{+}$absorption filaments revealed by ALMA are new. The BLA filaments in G0.253+0.016 are extraordinary because of their narrow minor axis dimension (less than $\sim 10^{4} \mathrm{AU}$ ), their $20 \mathrm{~km} \mathrm{~s}^{-1}$ width in absorption, and their low antenna temperatures. The more abundant NLA filaments are less extreme in their velocity extent. Comparison of the optically thick $\mathrm{HCO}^{+}$and $\mathrm{HCN}$ cubes with the other 


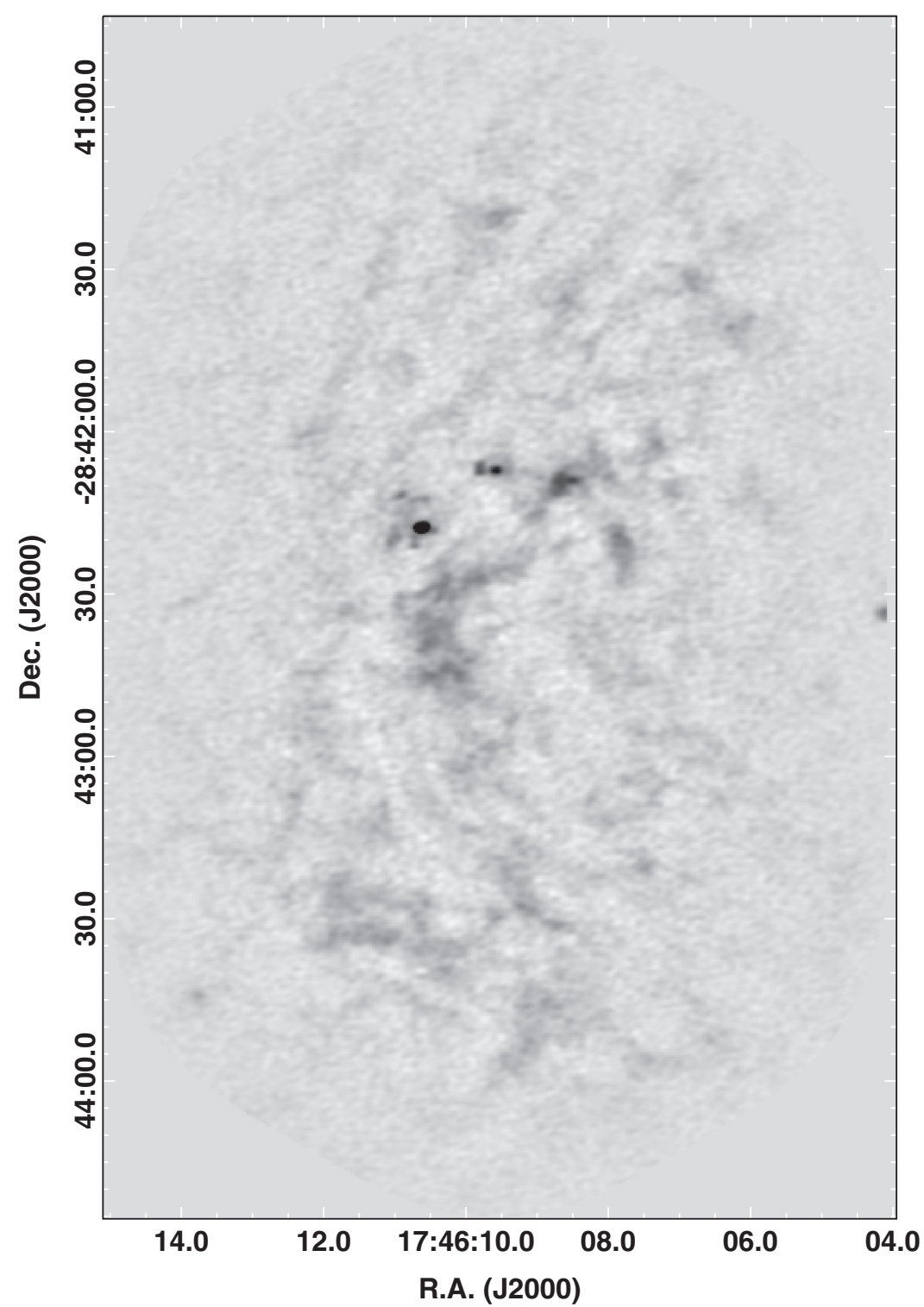

Figure 15. $3 \mathrm{~mm}$ continuum emission from G0.253+0.016. The bright point upper left of center is associated with the $\mathrm{H}_{2} \mathrm{O}$ maser source, located at $\mathrm{J} 2000=$ $17^{\mathrm{h}} 46^{\mathrm{m}} 10^{\mathrm{s}} 62,-28^{\circ} 42^{\prime} 17^{\prime \prime} .8$. This image and its interpretation is discussed in detail in Rathborne et al. (2014a).

ALMA spectral line cubes of optically thinner tracers such as $\mathrm{HNCO}, \mathrm{SiO}, \mathrm{C}_{2} \mathrm{H}, \mathrm{H}_{2} \mathrm{CS}, \mathrm{H}^{13} \mathrm{CN}$, and $\mathrm{H}^{13} \mathrm{CO}^{+}$show that $\mathrm{HCO}^{+}$ and $\mathrm{HCN}$ are heavily absorbed between $V_{\mathrm{LSR}}=-2.8$ and about $+30 \mathrm{~km} \mathrm{~s}^{-1}$. The BLAs are only seen in $\mathrm{HCO}^{+}$and have no corresponding features in any other species in either emission or absorption.

Two models are considered for the BLA filaments. The BLAs may be associated with dense gas in the Galactic center ring $(\mathrm{GCR})$ a portion of which has a radial velocity $V_{\mathrm{LSR}}=10$ to $30 \mathrm{~km} \mathrm{~s}^{-1}$ toward Galactic longitude of 0.18 (RodriguezFernandez et al. 2006; Rodriguez-Fernandez \& Combes 2008). Assuming that the GCR has similar orientation and kinematics but is larger than the $100 \mathrm{pc}$ radius ring proposed by Molinari et al. (2011), the front side is expected to have positive radial velocities while the far side is expected to have negative velocities. Given the possible association of G0.253+0.016 with the Molinari ring, the front side of GCR may be in front of G0.253+0.016. Low density, low-excitation gas could then be seen in absorption against the warmer emission from the cloud. In this scenario, the BLAs are produced by sub-thermal $\mathrm{HCO}^{+}$far in front (a few to as much as $\sim 200 \mathrm{pc}$ in front of $\mathrm{G} 0.253+0.016)$. Their narrow widths, highly supersonic velocity extents, and elongated filamentary morphology suggest that they are ram pressure confined. In this model, they may be caustic surfaces produced where shock fronts are moving in the plane of the sky and whose post-shock layers are sheets observed very close to edge-on. Thus, their depth along the line of sight may be much larger than their observed widths. The $\mathrm{HCO}^{+}$molecules must either survive passage through the shock, or are rapidly formed in the post-shock cooling layers. The crossing time of these filaments is about $10^{3}$ years. If molecule reformation is responsible for the $\mathrm{HCO}^{+}$abundance, then this timescale establishes a limit on the formation time.

The alternative model is that the BLAs are related to the non-thermal filaments near Galactic longitude 0.18 and are magnetically confined. This picture is motivated by the similar orientations of the BLAs and the $20 \mathrm{~cm}$ non-thermal filaments that lie only a few parsecs in projection from the southeast 
edge of G0.253+0.016. Both sets of filaments are oriented roughly at right angles to the Galactic plane. Using equipartition arguments applied to the synchrotron, Yusef-Zadeh \& Morris (1987) estimated that the magnetic fields in the non-thermal streamers have strengths of about $0.05-0.2 \mathrm{mG}$.

The polarization of the dust continuum emission from G0.253+0.016 has been measured at $\lambda=350 \mu \mathrm{m}$ (Dotson et al. 2010). The magnetic field is nominally orthogonal to the $350 \mu \mathrm{m}$ polarization vector. Inspection of Figure 39 in Dotson et al. (2010) shows that the magnetic field in the cloud interior where most of the sub-millimeter dust emission originates, closely follows the bow-shaped emission-line filaments observed in the optically thin tracers. The magnetic field in the northern part of $\mathrm{G} 0.253+0.016$ is approximately orthogonal to the Galactic plane and parallel to the BLA filaments. The magnetic field in the southern part of G0.253+0.016 is roughly aligned with the Galactic plane.

Application of the Chandrasekhar-Fermi method to estimating magnetic field strengths in the molecular gas in the CMZ as traced by sub-millimeter polarimetry of the dust continuum emission leads to field strength estimates of $1-3 \mathrm{mG}$ (Chuss et al. 2003). Using the scaling relations of magnetic field strength with cloud density based on Zeeman effect measurements (Crutcher 2012) and assuming a mean cloud density of few $\times 10^{5} \mathrm{~cm}^{-3}$ leads to similar field strength estimates. Ferrière (2010) presented a review of $\mathrm{CMZ}$ magnetic fields.

An ionization fraction of $\sim 10^{-7}$ (the estimated abundance of $\mathrm{HCO}^{+}$in the absorbing medium) implies very long ambipolar diffusion timescales and highly elongated structures if magnetic fields are present in the absorption region. Heavy molecular ions such as $\mathrm{HCO}^{+}$gyrate around the magnetic field lines at a frequency $f_{\text {gyro }} \sim e B / m\left(\mathrm{HCO}^{+}\right) c \approx 0.3 B_{\mathrm{mG}}$ radians per second. Here, $e$ is the electron charge, $c$ is the speed of light, $m_{\mathrm{HCO}+}=29 m_{\mathrm{H}}$ is the mass of an $\mathrm{HCO}^{+}$ molecule, and $B_{\mathrm{mG}}$ is the magnetic field in units of $1 \mathrm{mG}$. Using the observed spectral line half-width of the most prominent BLA filament, $\Delta V \sim 10 \mathrm{~km} \mathrm{~s}^{-1}$, implies a gyro-radius of $\mathrm{HCO}^{+}$molecules of $\sim 30 \mathrm{~km}$. The mean-free path of molecules, $\lambda_{m f p} \sim 1 / n\left(\mathrm{H}_{2}\right) \sigma$ for a density $n\left(\mathrm{H}_{2}\right)=10^{3}$ and a collision cross-section $\sigma \sim 10^{-15} \mathrm{~cm}^{-2}$ is $\lambda_{\text {mfp }} \sim 10^{12} \mathrm{~cm}$, five orders of magnitude smaller than the width of the BLA filaments $\left(\sim 10^{17} \mathrm{~cm}\right)$. The mean time between molecular collisions which lead to diffusion is $t_{\text {col }} \sim 10^{6} \mathrm{~s}$. To random-walk a distance $10^{5}$ times larger than the mean free-path requires about $10^{10}$ collisions. Thus, the time for molecules to diffuse over a distance of $r_{\text {filament }} \sim 10^{4}$ AU (the typical width of the absorption filaments) is therefore $t_{\mathrm{AD}} \sim 10^{10} t_{\mathrm{col}} \sim 10^{16} \mathrm{sec}$ onds. For the parameters implied by the observations, the magnetic field and the partially ionized molecular gas are tightly coupled. The effective diffusion velocity across the filaments is $V_{\text {perp }} \sim r_{\text {filament }} / t_{\mathrm{AD}} \sim 10 \mathrm{~cm} \mathrm{~s}^{-1}$. However, atoms and molecules can move unimpeded along the field lines at the $\Delta V \sim 10 \mathrm{~km} \mathrm{~s}^{-1}$ measured velocity dispersion. Thus the aspect ratio (length-to-width) of magnetized filaments can be as high as $\Delta V / V_{\text {perp }}>10^{5}$.

\subsection{Expansion of the Surface G0.253+0.016: Photo-ablation?}

Two mechanisms could be responsible for the apparent expansion of the near-side surface layers of G0.253+0.016; photo-ablation and stored magnetic energy. The first mechanism relies on external heating of the cloud surface layers by the combined effects of UV, soft X-ray, visual, and near-IR radiation that can photo-ablate cloud surfaces, generate large internal motions, and generate shocks which compress the cloud surface layers (Mellema et al. 2006; Henney et al. 2009; Arthur et al. 2011). Diffuse dust emitting between 8 and $70 \mu \mathrm{m}$ tends to be associated with photo-ionized plasmas in $\mathrm{H}$ II regions. The extended $20 \mathrm{~cm}$ free-free continuum (Yusefzadeh et al. 2004) and $8-70 \mu \mathrm{m}$ continuum emitted by diffuse mid-IR emission from warm dust implies that much of the inner $100 \mathrm{pc}$ of the Galaxy contains photo-ionized plasma and warm dust, likely produced by radiation from massive stars. Although the Spitzer and WISE mid-IR images show that the Galactic center bubble located between Sgr A and G0.253+0.016, and likely ionized and heated by the Arches and Quintuplet clusters, dominates the diffuse mid-IR emission in the CMZ, these images also exhibit somewhat fainter and more diffuse warm dust emission centered about $6^{\prime}$ ( $15 \mathrm{pc}$ in projection) due east of $\mathrm{G} 0.253+0.016$ at $[l, b]=[0.304,-0.067](\mathrm{J} 2000=17: 46: 36.2,-28: 42: 41)$.

Diffuse $20 \mathrm{~cm}$ radio continuum and several $\mathrm{H}$ II regions within $6^{\prime}$ of this region of peak infrared emission (but generally due east of $\mathrm{G} 0.253+0.016$ ) indicate the presence of OB stars whose energy output may irradiate and heat the G0.253+0.01 cloud's surface. If the Lyman continuum luminosity of these stars is $Q_{50} \sim 10^{50}$ ionizing photons per second, and located at a distance $D_{15}=15 \mathrm{pc}$, the electron density at the surface of G0.253+0.016 would be (neglecting any intervening extinction) about $n_{e}=\left(Q / 4 \pi \alpha_{B} r\right)^{1 / 2} D^{-1} \sim 40 Q_{50}^{1 / 2} r_{3}^{-1 / 2} D_{15}^{-1} \mathrm{~cm}^{-3}$ assuming a spherical cloud of radius $r=r_{3} \sim 3 \mathrm{pc}$ (here, $\alpha_{B}=2.6 \times 10^{-13} \mathrm{~cm}^{3} \mathrm{~s}^{-1}$ is the Case B hydrogen recombination coefficient). However, G0.253+0.016 appears filamentary with local radii of curvature at least an order of magnitude smaller in at least one direction. Thus, the actual electron density may be $n_{e}>10^{2} \mathrm{~cm}^{-3}$. The implied mass-loss rate due to heating (assuming that the gas is accelerated to about the soundspeed in ionized gas; $\sim 10 \mathrm{~km} \mathrm{~s}^{-1}$ ) is about $10^{-3} M_{\odot} \mathrm{yr}^{-1}$ or only about $10^{2}$ to $10^{3} M_{\odot}$ in $0.25 \mathrm{Myr}$. This is an order of magnitude lower than the mass-loss rate estimated from the NLA absorption filaments. Unless additional energy input mechanisms operate, photo-ablation is an unlikely source of cloud surface layer expansion.

\subsection{Expansion of the Surface G0.253+0.016: Magnetic Bounce?}

Longmore et al. (2013) proposed that G0.253+0.016 passed near Sgr A about 0.25 Myr ago. Passage of the cloud through the deepest part of the Galactic gravitational potential would have compressed the cloud. If its interior magnetic fields were sufficiently tangled, they would act as a spring, storing the energy of compression, limiting the dissipation of internal motions by shocks. As G0.253+0.016 emerged from the potential well, the compressed fields could drive re-expansion of the cloud. While some regions with high density or mass-to-flux ratios may collapse, less dense or relatively weakly bound parts of the cloud such as its outer layers, may expand as the fields relax.

An order of magnitude estimate of the mean field strength required to support such a "bounce" can be obtained by assuming that the stored magnetic energy, $E_{B} \sim f B^{2} r^{3}$ is comparable to the gravitational self-energy $E_{G} \sim g G M^{2} / r$. Here, $f$ and $g$ are factors of the order of unity depending on the magnetic field geometry, the cloud shape, and density distribution, and for G0.253+0.016, $r \sim 3 \mathrm{pc}$ is the mean cloud radius, and $M \sim 10^{5} M_{\odot}$ is the cloud mass. If the gravitational potential energy is similar to the magnetic energy (as required by a "bounce"), these parameters imply a mean magnetic field strength $B \sim 1.5 \mathrm{mG}$, consistent with the 
magnetic field-strength estimates. Thus, the adiabatic behavior of a tangled internal magnetic field could drive a re-expansion of $\mathrm{G} 0.253+0.016$ following its emergence form the deepest part of the Galactic gravitational potential.

Future work should search for evidence for BLAs in other optically thick ground-state transitions of ions such as $\mathrm{N}_{2} \mathrm{H}^{+}$. BLAs detected only in other ions would lend support to the magnetic structure interpretation. BLAs detected in other neutral species, especially ones with enhanced abundances in shocks, would provide support for the shock-wave interpretation. High-resolution dust continuum polarization measurements and searches for Zeeman splitting are needed to constrain the magnetic field geometry and strength to determine if the magnetic flux-to-mass ratio is super-critical and allows the cloud to collapse, or sub-critical and may prevent collapse and formation of a cluster of stars.

\section{CONCLUSIONS}

ALMA Cycle 0 observations of the massive, compact, and dense Galactic center cloud, G0.253+0.016 are presented with an angular resolution of about $1^{\prime \prime}$.7 in the $\mathrm{HCO}^{+}$and $\mathrm{H}^{13} \mathrm{CO}^{+} J=1-0$ lines. The main results derived from these observations are as follows.

1. The observed intensity ratios of $\mathrm{HCO}^{+}$and $\mathrm{H}^{13} \mathrm{CO}^{+}$indicate that the mean optical depth of $\mathrm{HCO}^{+}$is around unity or higher. The observed $J=1-0 \mathrm{HCO}^{+}$brightness temperature of $2-12 \mathrm{~K}$ in a cloud in which the kinetic temperature of the gas is estimated to be around $60-80 \mathrm{~K}$ indicates sub-thermal excitation at the $\mathrm{HCO}^{+}$photosphere and an $\mathrm{H}_{2}$ density $\left(n\left(\mathrm{H}_{2}\right) \sim 10^{3} \mathrm{~cm}^{-3}\right)$ more than an order of magnitude lower than the critical density for the $J=1-0$ transition and more than an order of magnitude lower than the mean density of $\mathrm{G} 0.253+0.016$.

2. The ALMA observations reveal a network of blueshifted (relative to the cloud) absorption filaments seen against the sub-thermally excited $\mathrm{HCO}^{+}$emission. These filaments must have densities even lower than density at the $\tau_{12} \sim 1$ photosphere where most of the $\mathrm{HCO}^{+}$emission is produced. A few BLA filaments have line widths of over $20 \mathrm{~km} \mathrm{~s}^{-1}$, widths of only a few arc seconds, and lengths of nearly 1 arcmin. They are only seen in $\mathrm{HCO}^{+}$. The BLA filaments may be foreground magnetic structures possibly associated with the brightest non-thermal filaments that cross the Galactic plane at longitude 0.18 . Alternatively, they may be shock fronts in foreground CMZ clouds propagating orthogonal to the line of sight so that their compressed layers are seen nearly edge-on with a depth along the line of sight much greater than their projected widths. Additionally, G0.253+0.016 is laced with dozens of NLA filaments with line widths of less than $20 \mathrm{~km} \mathrm{~s}^{-1}$. Most of these features are seen on the blueshifted side of the line profiles. A few have counterparts in $\mathrm{HCN}$. Some absorption filaments seen in both $\mathrm{HCO}^{+}$and $\mathrm{HCN}$ which extend over faint $\mathrm{HCN}$ backgrounds go into emission in $\mathrm{HCN}$ but not in $\mathrm{HCO}^{+}$. $\mathrm{HCN}$ also shows extensive absorption on the blueshifted side of $\mathrm{G} 0.253+0.016$. These characteristics suggest that the NLA filaments trace sub-thermally excited, optically thick gas on the front surface of G0.253+0.016.

3. The blueshift of the absorption filaments relative to the peak $\mathrm{HCO}^{+}$emission indicates that the surface layers of $\mathrm{G} 0.253+0.016$ are expanding with velocities larger than $5-20 \mathrm{~km} \mathrm{~s}^{-1}$ with respect to the cloud's center of mass. The apparent "P-Cygni" line profile may be an indication of reexpansion of the G0.253+0.016 surface layers following a recent passage close to $\mathrm{Sgr}$ A. Compression of a milliGauss magnetic field during the passage, followed by subsequent expansion driven by stored magnetic energy may be responsible.

4. Weather or not $\mathrm{G} 0.253+0.016$ will form a star cluster or only a few stars remains unclear. Although the $\mathrm{HCO}^{+}$absorption indicates that the cloud surface layers are expanding, the cloud interior may be in the earliest stages of star formation as indicated by the presence of at least one cloud core with an associated water maser. The dynamic state of the dozens of other $3 \mathrm{~mm}$ dust condensations in $\mathrm{G} 0.253+0.016$ remains unclear. Measurements of the amplitude and geometry of the magnetic field are needed to determine if the magnetic pressure is sufficient or insufficient to prevent this massive, high-density, and compact cloud from collapsing to form a cluster or association of stars.

This research of J.B. and A.G. was in part supported by National Science Foundation (NSF) grant AST-1009847. Observations reported here were obtained at the Atacama Large Millimeter Array (ALMA) located in Chile. The authors acknowledge our ALMA Contact Scientist, Dr Crystal Brogan, for assistance in preparing the observations and performing the initial data calibration. J.M.R acknowledges funding support via CSIRO's Julius Career Award that was used to host a weeklong team meeting which laid the foundation for this work. J.M.R, S.N.L, J.M.D.K, J.B., and N.B. acknowledge the hospitality of the Aspen Center for Physics, which is supported by the National Science Foundation grant No. PHY-1066293. This paper makes use of the following ALMA data: ADS/ JAO.ALMA\#2011.0.00217.S. ALMA is a partnership of the European Southern Observatory (ESO) representing member states, Associated Universities Incorporated (AUI) and the National radio Astronomy Observatories (NRAO) for the National Science Foundation (NSF) in the USA, NINS in Japan, NRC in Canada, and NSC and ASIAA in Taiwan, in cooperation with the Republic of Chile. The Joint ALMA Observatory (JAO) is operated by ESO (Europe), AUI/NRAO (USA), and NAOJ (Japan). The National Radio Astronomy Observatory is a facility of the National Science Foundation operated under cooperative agreement by Associated Universities, Inc.

\section{REFERENCES}

Ao, Y., Henkel, C., Menten, K. M., et al. 2013, A\&A, 550, A135

Arendt, R. G., Stolovy, S. R., Ramírez, S. V., et al. 2008, ApJ, 682, 384 Arthur, S. J., Henney, W. J., Mellema, G., de Colle, F., \& Vázquez-Semadeni, E. 2011, MNRAS, 414, 1747

Athanassoula, E. 1992a, MNRAS, 259, 328

Athanassoula, E. 1992b, MNRAS, 259, 345

Bally, J., Aguirre, J., Battersby, C., et al. 2010, ApJ, 721, 137

Bally, J., \& Langer, W. D. 1982, ApJ, 255, 143

Bally, J., Langer, W. D., Stark, A. A., \& Wilson, R. W. 1987a, ApJL, 312, L45 Bally, J., Stark, A. A., Wilson, R. W., \& Henkel, C. 1987b, ApJS, 65, 13 Bally, J., Stark, A. A., Wilson, R. W., \& Henkel, C. 1988, ApJ, 324, 223 Binney, J., Gerhard, O. E., Stark, A. A., Bally, J., \& Uchida, K. I. 1991, MNRAS, 252, 210

Breen, S. L., \& Ellingsen, S. P. 2011, MNRAS, 416, 178

Chuss, D. T., Davidson, J. A., Dotson, J. L., et al. 2003, ApJ, 599, 1116 Clark, P. C., Glover, S. C. O., Ragan, S. E., Shetty, R., \& Klessen, R. S. 2013, ApJL, 768, L34

Contopoulos, G., \& Papayannopoulos, T. 1980, A\&A, 92, 33

Crutcher, R. M. 2012, ARA\&A, 50, 29

Dotson, J. L., Vaillancourt, J. E., Kirby, L., et al. 2010, ApJS, 186, 406

Ferrière, K. 2010, AN, 331, 27 
Ferrière, K., Gillard, W., \& Jean, P. 2007, A\&A, 467, 611

Figer, D. F., Najarro, F., Gilmore, D., et al. 2002, ApJ, 581, 258

Foster, J. B., Jackson, J. M., Barnes, P. J., et al. 2011, ApJS, 197, 25

Foster, J. B., Rathborne, J. M., Sanhueza, P., et al. 2013, PASA, 30, 38

Goto, M., Usuda, T., Geballe, T. R., et al. 2011, PASJ, 63, L13

Goto, M., Usuda, T., Nagata, T., et al. 2008, ApJ, 688, 306

Habibi, M., Stolte, A., Brandner, W., Hußmann, B., \& Motohara, K. 2013, A\&A, 556, A26

Henney, W. J., Arthur, S. J., de Colle, F., \& Mellema, G. 2009, MNRAS, 398,157

Higuchi, A. E., Chibueze, J. O., Habe, A., Takahira, K., \& Takano, S. 2014, AJ, 147,141

Jackson, J. M., Rathborne, J. M., Foster, J. B., et al. 2013, PASA, 30, 57

Kauffmann, J., Pillai, T., \& Zhang, Q. 2013, ApJL, 765, L35

Langer, W. D., \& Penzias, A. A. 1990, ApJ, 357, 477

Lis, D. C., \& Carlstrom, J. E. 1994, ApJ, 424, 189

Lis, D. C., \& Menten, K. M. 1998, ApJ, 507, 794

Lis, D. C., Menten, K. M., Serabyn, E., \& Zylka, R. 1994, ApJL, 423, L39

Lis, D. C., Serabyn, E., Zylka, R., \& Li, Y. 2001, ApJ, 550, 761

Liszt, H., \& Lucas, R. 2004, A\&A, 428, 445

Longmore, S. N., Kruijssen, J. M. D., Bally, J., et al. 2013, MNRAS, 433, L15

Longmore, S. N., Rathborne, J., Bastian, N., et al. 2012, ApJ, 746, 117

Lyons, J. R., \& Young, E. D. 2005, Natur, 435, 317

Mellema, G., Arthur, S. J., Henney, W. J., Iliev, I. T., \& Shapiro, P. R. 2006, ApJ, 647,397

Menten, K. M., Pillai, T., \& Wyrowski, F. 2005, in IAU Symp. 227, Massive Star Birth: A Crossroads of Astrophysics, ed. R. Cesaroni, M. Felli, E. Churchwell, \& M. Walmsley (Cambridge: Cambridge Univ. Press), 23
Milam, S. N., Savage, C., Brewster, M. A., Ziurys, L. M., \& Wyckoff, S. 2005, ApJ, 634, 1126

Mills, E. A. C., \& Morris, M. R. 2013, ApJ, 772, 105

Molinari, S., Bally, J., Noriega-Crespo, A., et al. 2011, ApJL, 735, L33

Molinari, S., Swinyard, B., Bally, J., et al. 2010a, A\&A, 518, L100

Molinari, S., Swinyard, B., Bally, J., et al. 2010b, PASP, 122, 314

Morris, M., \& Serabyn, E. 1996, ARA\&A, 34, 645

Pierce-Price, D., Richer, J. S., Greaves, J. S., et al. 2000, ApJL, 545, L121

Portegies-Zwart, S. F., McMillan, S. L. W., \& Gieles, M. 2010, ARA\&A, 48, 431

Ramírez, S. V., Arendt, R. G., Sellgren, K., et al. 2008, ApJS, 175, 147

Rathborne, J. M., Longmore, S. N., Jackson, J. M., et al. 2014a, ApJ, in press (arXiv:1409.0935)

Rathborne, J. M., Longmore, S. N., Jackson, J. M., et al. 2014b, ApJ, 786,140

Riquelme, D., Amo-Baladrón, M. A., Martín-Pintado, J., et al. 2010, A\&A, 523, A51

Rodriguez-Fernandez, N. J., \& Combes, F. 2008, A\&A, 489, 115

Rodriguez-Fernandez, N. J., Combes, F., Martin-Pintado, J., Wilson, T. L., \& Apponi, A. 2006, A\&A, 455, 963

Röllig, M., \& Ossenkopf, V. 2013, A\&A, 550, A56

van der Tak, F. F. S., Black, J. H., Schöier, F. L., Jansen, D. J., \& van Dishoeck, E. F. 2007, A\&A, 468, 627

Yusef-Zadeh, F., Cotton, W., Viti, S., Wardle, M., \& Royster, M. 2013, ApJL, 764, L19

Yusef-Zadeh, F., Hewitt, J. W., \& Cotton, W. 2004, ApJS, 155, 421

Yusef-Zadeh, F., \& Morris, M. 1987, ApJ, 322, 721

Yusefzadeh, F., Hewitt, J. W., \& Cotton, W. 2004, ApJS, 155, 42 